\title{
Microphysiological Systems for Studying Cellular Crosstalk During the Neutrophil Response to Infection
}

\section{OPEN ACCESS}

Edited by:

Felix Ellett,

Massachusetts General Hospital and Harvard Medical School, United States

Reviewed by:

Caroline N. Jones,

The University of Texas, United States

Francis Lin

University of Manitoba, Canada

Steven L. Raymond,

University of Florida, United States

${ }^{*}$ Correspondence:

Laurel E. Hind

laurel.hind@colorado.edu

${ }^{\dagger}$ These authors have contributed equally to this work and share first authorship

Specialty section:

This article was submitted to Molecular Innate Immunity, a section of the journal

Frontiers in Immunology

Received: 30 January 2021 Accepted: 09 April 2021 Published: 27 April 2021

Citation:

Richardson IM, Calo CJ and Hind LE (2021) Microphysiological Systems for Studying Cellular Crosstalk During the Neutrophil Response to Infection.

Front. Immunol. 12:661537. doi: 10.3389/fimmu.2021.661537

\author{
Isaac M. Richardson ${ }^{\dagger}$, Christopher J. Calo ${ }^{\dagger}$ and Laurel E. Hind ${ }^{*}$ \\ Department of Chemical and Biological Engineering, University of Colorado - Boulder, Boulder, CO, United States
}

Neutrophils are the primary responders to infection, rapidly migrating to sites of inflammation and clearing pathogens through a variety of antimicrobial functions. This response is controlled by a complex network of signals produced by vascular cells, tissue resident cells, other immune cells, and the pathogen itself. Despite significant efforts to understand how these signals are integrated into the neutrophil response, we still do not have a complete picture of the mechanisms regulating this process. This is in part due to the inherent disadvantages of the most-used experimental systems: in vitro systems lack the complexity of the tissue microenvironment and animal models do not accurately capture the human immune response. Advanced microfluidic devices incorporating relevant tissue architectures, cell-cell interactions, and live pathogen sources have been developed to overcome these challenges. In this review, we will discuss the in vitro models currently being used to study the neutrophil response to infection, specifically in the context of cell-cell interactions, and provide an overview of their findings. We will also provide recommendations for the future direction of the field and what important aspects of the infectious microenvironment are missing from the current models.

Keywords: neutrophil, microfluidics, infection, in vitro models, innate immunity, cell-cell interactions, inflammation, antimicrobial functions

\section{INTRODUCTION}

The innate immune response to infection is a complicated process requiring a coordinated effort by many cell populations. Neutrophils are one of the first cells to arrive at an infection and are critical in limiting pathogen spread, but their response must be tightly regulated. Defects in neutrophil recruitment can lead to recurrent, unresolved infections and excessive neutrophil activity can lead to chronic inflammation and tissue damage; therefore, understanding the signals driving neutrophil recruitment is critical for controlling their response following an infection. It has been shown that the tissue environment and cellular interactions can have a significant impact on neutrophil function but new ways of studying the innate immune system are needed to determine how these interactions affect neutrophil function.

Two experimental systems are predominantly used for studying the innate immune response to infection: in vitro "cells-in-a-dish" and in vivo animal models. While animal models allow researchers to visualize the neutrophil response in a physiologically relevant environment that includes cellular interactions, their inherent complexity makes teasing apart the role of specific 
signals or interactions difficult. Therefore, in vitro models where primary human cells can be used are a necessary complement to animal models. Unfortunately, traditional in vitro systems lack important components of the infectious microenvironment including cell-cell interactions, three-dimensional structures, and a tissue mimic or extracellular matrix. Recently, several physiologically relevant in vitro models have been developed that incorporate these features. These studies have demonstrated the importance of including relevant geometries, cell-cell interactions, and cell matrix interactions in investigating the immune response.

\section{THE NEUTROPHIL RESPONSE TO INFLAMMATION}

As the first cellular responders during inflammation, neutrophils serve as the foot soldiers of the innate immune response. Within minutes of infection or injury, neutrophils activate and migrate from the blood vessel to the site of inflammation where they clear pathogens and signal for the activation and recruitment of other immune cells. To achieve this rapid response, neutrophils employ non-specific antimicrobial activities, such as the generation of reactive oxygen species (ROS), that damage both pathogenic and host cells. Consequently, excessive neutrophil activity contributes to the pathology of several inflammatory diseases, including rheumatoid arthritis and gout $(1,2)$. Conversely, insufficient neutrophil activity is associated with recurrent and more severe infections as encountered by individuals with neutropenia, chronic granulomatous disease, and leukocyte adhesion deficiency (3-5). Therefore, it is crucial that neutrophil activity is highly regulated to prevent excessive collateral damage of healthy tissue, while still protecting the host from pathogens.

Neutrophil recruitment to inflammation is mediated by an intricate meshwork of cellular interactions including, but not limited to, interactions between neutrophils, endothelial cells, other immune cells, and pathogens (6-10). Following infection, neutrophils escape the circulation through a series of interactions with endothelial cells, which line the blood vessel lumen, in a process known as the leukocyte adhesion cascade (Figure 1). Upon activation by signals from infected or injured tissue, blood vessel endothelial cells upregulate selectins, which then bind and capture circulating neutrophils $(11,12)$. These neutrophils then roll along the endothelium, accumulating bonds between integrins on their surface and adhesion molecules on the endothelial surface. Eventually, the neutrophils stop rolling and begin crawling along the endothelium, extending protrusions in search of a point to extravasate, or migrate, through the blood vessel $(13,14)$. Extravasation, whether transcellular (through an endothelial cell) or paracellular (between two endothelial cells), is mediated by the binding of adhesion molecules on the endothelial cell surface and their corresponding neutrophil ligands (14-18). After extravasation, neutrophils release neutrophil elastase to make a hole in the basement membrane of the blood vessel and migrate into the tissue (19). While this process is primarily governed by neutrophil-endothelial cell interactions, other cell types, such as monocytes, have been shown to influence neutrophil extravasation (20).

Once in the tissue, neutrophils follow chemoattractant gradients to locate and migrate to the site of inflammation. Neutrophils have over 30 receptors on their surface that recognize proinflammatory signals including cytokines released by tissue resident cells, damageassociated molecular patterns (DAMPs), and pathogen-associated molecular patterns (PAMPs) that guide their movement (21) (Figure 1). DNA, high mobility group protein B1, N-formyl peptides, extracellular matrix proteins, adenosine triphosphate (ATP), and uric acid are examples of DAMPs that drive early neutrophil recruitment in tissue. Their roles in this process have been previously reviewed (22-24). Tissue resident cells, such as macrophages and mast cells, also recognize DAMPs, PAMPs, and inflammatory cytokines. Upon stimulation, these resident cells produce and secrete inflammatory cytokines of their own, including interleukin-8 (IL-8), a potent neutrophil chemoattractant (25-28). As chemoattractants populate the local environment, they establish a gradient that directs neutrophils to the site of inflammation (29-32).

At the site of inflammation, neutrophils begin a neutrophilrecruitment feedback loop by releasing interleukin-1 $\beta$ (IL-1 $\beta$ ), which activates macrophages, dendritic cells, $\gamma \delta \mathrm{T}$ cells, and endothelial cells to produce and release chemokines to recruit more neutrophils $(33,34)$. In addition to inflammatory cytokines, tissue resident cells and migrating neutrophils produce lipid mediators, most notably leukotriene $\mathrm{B}_{4}\left(\mathrm{LTB}_{4}\right)$, major contributors to a sustained neutrophil response (35-40). In both sterile and infected neutrophil responses, neutrophils swarm at the site of inflammation. Swarming is a process whereby neutrophils cluster around necrotic tissue and pathogens, including bacteria, fungi, and parasites (41). At the inflammatory site neutrophils continue to secrete $\mathrm{LTB}_{4}$ and express integrins to facilitate swarming. These swarms form a seal encased by late recruited macrophages and monocytes (42).

Upon reaching the site of inflammation, neutrophils employ various antimicrobial techniques to clear cellular debris as well as contain and kill pathogens. Their primary method of pathogen clearance is engulfment or phagocytosis $(43,44)$. Additionally, neutrophils attack pathogens by releasing ROS during respiratory bursts $(45-47)$ and producing neutrophil extracellular traps (NETs) (48-50). NETs consist of decondensed neutrophil DNA and associated proteins in a web-like structure that prevent pathogens from spreading, while marking them for phagocytosis $(48,49)$.

As is the case with extravasation, neutrophil antimicrobial activity is influenced by interactions with other cells (50-52). Additionally, ROS and NETs contain proinflammatory signals that activate other cells, including neutrophils, to sustain and increase the inflammatory response. If not properly regulated, these signals can cause unwanted autoimmune responses and disease (53-56). Pathogens also influence neutrophil antimicrobial activity (57). For example, whether or not a neutrophil produces NETs is partially determined by the size of microbes (58). 


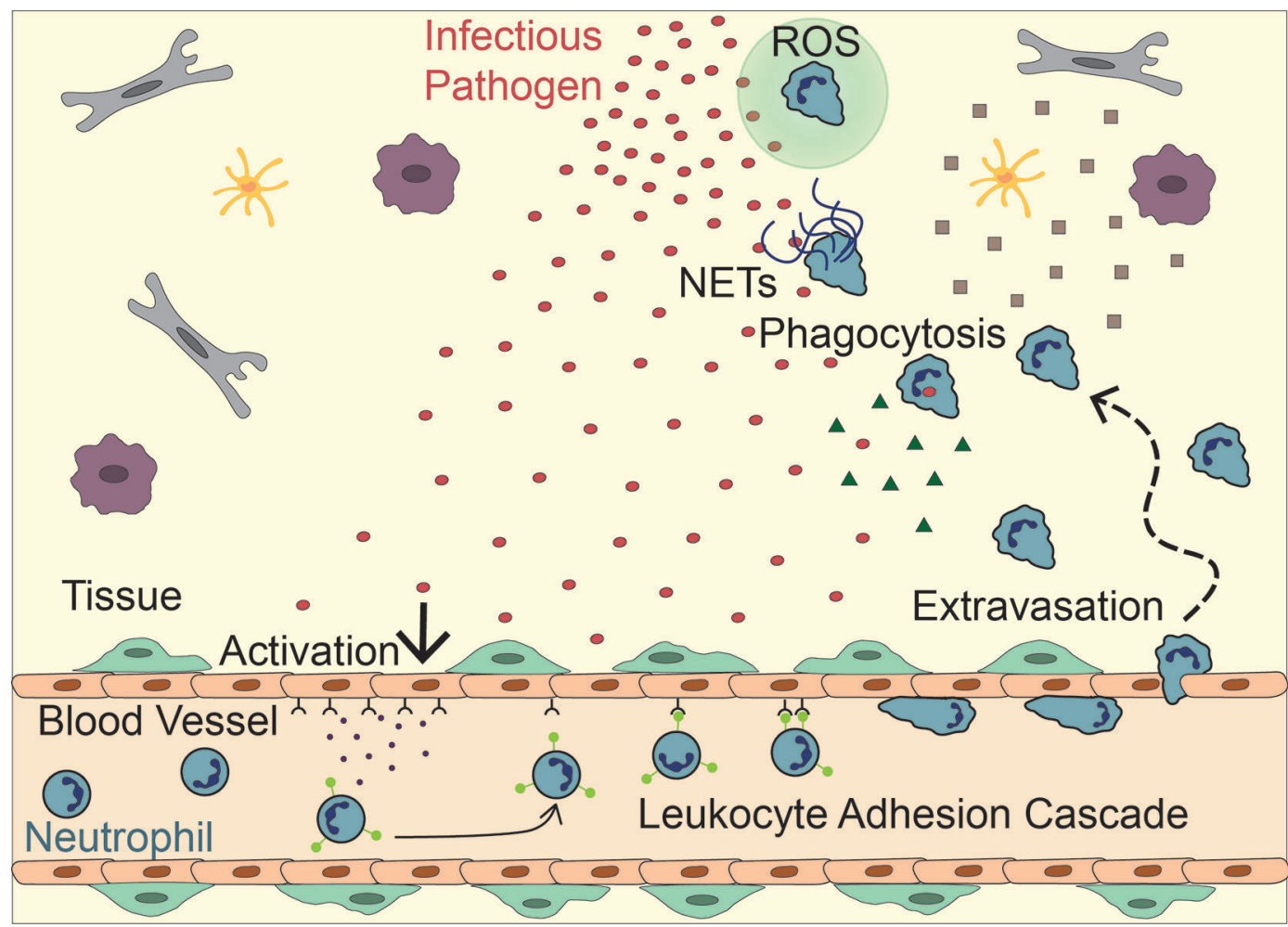

Cellular Components

Neutrophil Macrophage Dendritic Cell Fibroblast Endothelial Cell Pericytes

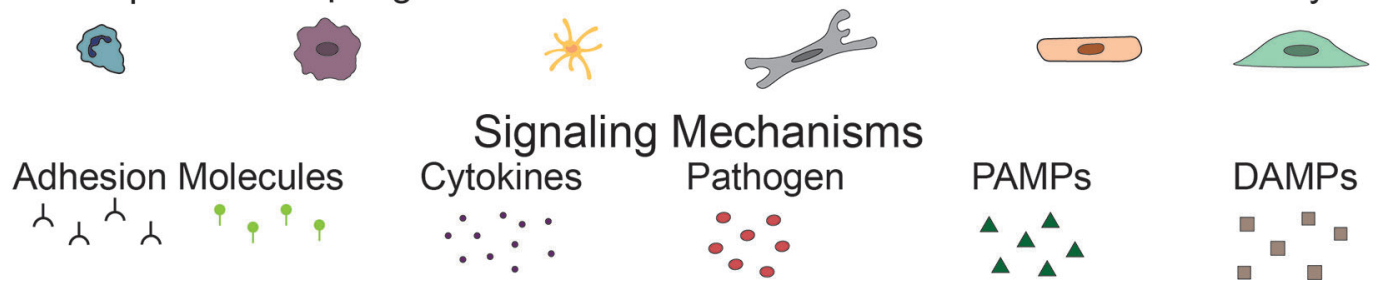

FIGURE 1 | Neutrophil Response to Infection. Following infection, endothelial cells lining the vasculature become activated, releasing adhesion molecules and cytokines. These signals activate neutrophils, initiating the leukocyte adhesion cascade. Neutrophils then extravasate through the blood vessel and migrate to the site of infection following PAMPs, released by the pathogen, and DAMPs released by tissue resident cells (macrophages, dendritic cells, fibroblasts). There, they fight the infection by releasing NETs and Reactive Oxygen Species (ROS), and directly phagocytosing the pathogen.

Interaction with macrophages is especially important in the regulation of both neutrophil recruitment and resolution. Monocyte-derived macrophages clear NETs to prevent an overactive immune response, resolving inflammation (59). Dysregulation of this process, namely in the case of Lupus macrophages, leads to inflammasome activation in response to NETs, which in turn triggers the release of inflammatory cytokines inducing further NET production, or NETosis (60). Additionally, tissue resident macrophages downregulate neutrophil swarming and activity by hiding microlesions from neutrophils in tissue (61). Macrophages also inhibit sustained neutrophil recruitment and neutrophil-mediated killing in fungal infections by preventing fungal germination, a process that activates neutrophils (62). Finally, macrophages play a role in phagocytosis of neutrophils and their components, helping to resolve inflammation $(63,64)$.

To better understand the neutrophil response to infection, regulatory interactions between neutrophils and other cells, both host and pathogenic, must be studied in experimental systems that account for cell-cell interactions. While early studies with simple in vitro and complex in vivo models have gleaned fundamental knowledge of these interactions, they have key limitations. More recently, a range of physiologically relevant microfluidic devices have been developed to circumvent these limitations. These devices give a controlled, highly tailorable environment with low cost and high throughput for the study of human immune cell interactions. This review will focus on in vitro devices and how they have helped elucidate the effects of 
neutrophil interactions with host and pathogenic cells on the neutrophil response.

\section{IN VITRO MODELS FOR STUDYING THE NEUTROPHIL RESPONSE}

Historically, studies investigating the role of neutrophils in the immune response have been conducted in two types of models: simple in vitro systems, primarily investigating isolated interactions between two cell populations or a single cell population and an activating signal, and complex in vivo models, such as zebrafish or mice. These systems have led to important findings that serve as a knowledge base for the field; however, they have limitations. Simple in vitro models fail to capture the three-dimensional architecture of an in vivo environment along with pertinent physical cues. Furthermore, isolating cell types and investigating their interactions neglects important cellular signals from their environment. Conversely, animal models provide a complex and physiologically relevant three-dimensional environment to study immune responses; however, they are costly, low throughput, have a high degree of variability, and do not always translate to the human immune response. As such, recent efforts have focused on developing new experimental platforms for studying the neutrophil response to infection that include the physiological relevance of in vivo systems while preserving the advantages of studying cells in vitro.
Traditionally, simple in vitro models, such as Transwell assays and Dunn chambers, have been used to study neutrophils in twodimensional environments. The first experiments investigating cell-cell interactions and the neutrophil response to infection used a simple Transwell assay. In general, these models consist of a well-in-well system in which endothelial monolayers are formed on porous membranes in the top well and inflammatory signals are added into the bottom well (Figure 2A). Neutrophils are then added to the top well and the number of neutrophils that migrate through the monolayer into the bottom well is quantified (65). This type of device has been used to model the innate immune response in different environments by altering the vascular cell sources and varying activation signals (66-77). For example, these devices have been used to study neutrophil migration in response to common inflammatory chemoattractants, including IL-8 and $\mathrm{N}$ formylmethionine-leucyl-phenylalanine (fMLP) (29, 78) (Figure $\mathbf{2 A}$, top), and in response to live bacterial infections, including Escherichia coli, Streptococcus pneumoniae, and Staphylococcus aureus (79) (Figure 2A, bottom). They have also been used to study the neutrophil response in the presence of additional supporting cell types such as pericytes $(80,81)$. Transwell assays are well designed for use in investigating overall neutrophil migratory behavior and their interaction with an endothelium, but, due to their design, they only allow for end-point analysis. Therefore, more complex models are required for real time monitoring of neutrophil behavior.

Animal models, primarily mice and zebrafish, have been the standard for conducting studies in complex, physiologically
A

Transwells

Chemokines
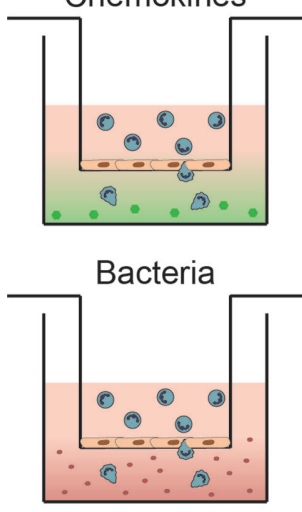

B

2D Microfluidic Devices

Neurophil Migration

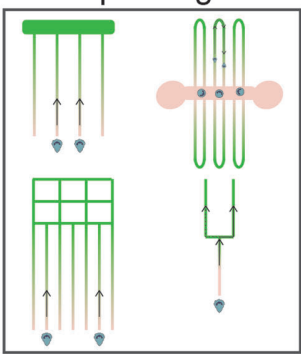

Interaction with Pathogens

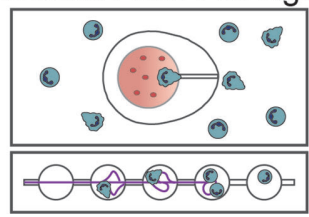

C

3D Microfluidic Devices

Side View
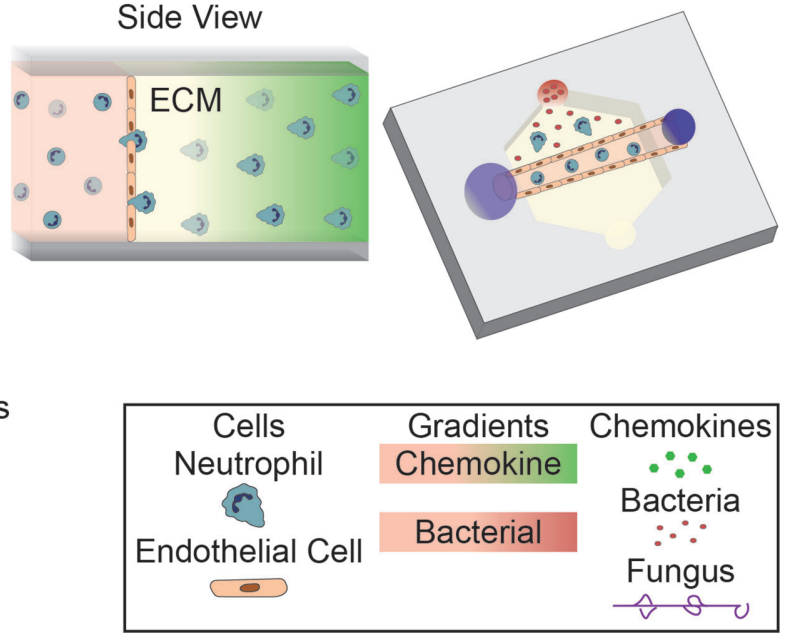

FIGURE 2 | In Vitro Systems for Studying the Neutrophil Response to Infection. (A) Transwell assays, a well-in-well system with a porous membrane divider, are used to investigate neutrophil migration to chemokines (top, green gradient) and bacterial sources (bottom, red gradient) through cellular monolayers. (B) 2D microfluidic devices are used to investigate various aspects of neutrophil migration, including neutrophil reverse migration and migration through bifurcations, to soluble chemokines (top). Devices have also been designed to investigate direct interactions between neutrophils and both bacterial and fungal pathogens (bottom). (C) 3D microfluidic devices are used to investigate neutrophil migration to soluble chemokines in an extracellular matrix hydrogel following extravasation through an endothelium. Neutrophils migrate through an endothelial monolayer, seeded on the hydrogel, and into the ECM. (D) Organotypic microfluidic devices include a model vasculature containing endothelial cells in a relevant lumen geometry. These devices use both chemokines and live pathogens to induce migration. 
relevant systems $(82,83)$. Unlike Transwell assays, these animal models allow for in vivo imaging of neutrophils in a complex environment. In addition to live imaging of neutrophils, animal models have been used to identify complex neutrophil activity not seen in simple in vitro models, such as NETosis (48), migration away from a wound following interaction with a macrophage (84), and reverse transendothelial migration (85), the process whereby extravasated or partially extravasated neutrophils migrate back through the endothelium to renter the blood vessel. While these models allow for live imaging and can capture complex neutrophil phenomena, they do not always translate to human neutrophil activity. Furthermore, their inherent complexity makes it difficult to isolate and investigate the role of specific cell-cell interactions in regulating the neutrophil response. Consequently, recent work has focused on developing physiologically relevant in vitro microfluidic devices that combine the advantages of simple in vitro models while mimicking the in vivo environment.

Microfluidic devices are well-suited for studying the immune response because they can be designed with customizable geometries and require low reagent and cell volumes. These factors make them ideal for working with primary human cells and for precisely controlling the spatiotemporal presentation of signaling molecules and pathogens. During their response in vivo, neutrophils are exposed to activating signals from both tissue resident cells and the pathogen at different times and locations; therefore, spatiotemporal resolution of signal presentation is critical for modeling the in vivo environment and developing an understanding of how neutrophils respond to these varying signals. Additionally, they are straightforward to create and use, cost-effective, and can be multiplexed for highthroughput studies. These devices can be designed to include cellular components, tissue components, and architectures relevant for modeling the human immune system. Furthermore, they can be created to monitor innate immune cell function through many mechanisms including time-lapse imaging of cell behavior, collection and evaluation of cells, and quantification of soluble signals. Importantly, microfluidic devices allow for single-cell analysis which is of particular interest for studying neutrophils as recent reports have found high levels of neutrophil heterogeneity (86). Recently, researchers have developed increasingly complex devices incorporating physiologically relevant components and structures. These devices have brought about new insights into the role of both tissue architecture and neutrophil interactions with tissue components, vascular and stromal cells, and pathogens in modulating the innate immune response.

Several groups have developed microfluidic devices that allow for real time imaging and evaluation of the neutrophil response. These devices have been specifically designed to include elements that allow them to investigate individual neutrophil functions. Microfluidic devices have been designed for studying mechanisms of neutrophil migration (87-91), NETosis (9294), ROS generation $(92,95)$ and, more recently, direct interactions between neutrophils and pathogens (Figure 2B). While many devices use representative chemokines to model an infectious source, devices have recently been designed to incorporate live, intact bacteria and fungi (Figure 2B, bottom). The addition of live pathogens increases the relevance of the neutrophil response and allows researchers to investigate the direct mechanisms of interaction between neutrophils and pathogens. A few recently described two-dimensional models for investigating neutrophil interactions with Aspergillus fumigatus (A. fumigatus) follow a similar design. The different models all have a chamber for loading of fungal conidia, space for outward hyphal growth to occur, and a separate loading port for the introduction of neutrophils. One device was designed to investigate the influence of directed migration on neutrophil interactions with the fungus $A$. fumigatus by requiring neutrophils to migrate up an fMLP gradient into a chemotaxis chamber before interacting with fungal spores (96). Devices have also been developed to investigate other neutrophil functions following interaction with fungal pathogens including one containing an array of fungal clusters designed to monitor neutrophil swarming (97). In addition to fungal pathogens, devices have been designed to investigate neutrophilic response to bacteria. A device of this nature was developed with 'war theaters' consisting of an inner microchamber seeded with bacteria inside a larger chamber seeded with neutrophils, allowing researchers to study the neutrophil recruitment and interactions with growing bacteria (Figure 2B, bottom) (98). These microfluidic devices have allowed researchers to investigate important components of the neutrophil response in an effective, high-throughput, and easily repeatable way and have led to critical discoveries about neutrophil-pathogen interactions that could only be studied in highly controlled environments. However, these devices do not account for key components of the in vivo infectious microenvironment, including three-dimensional structures, and do not replicate important events during the neutrophil response to infection such as extravasation through the vasculature. Therefore, devices with additional complexity have been developed to investigate the influence of those factors.

It has become apparent that multicellular interactions have a significant impact on the neutrophil response to infection. Additionally, migration in a three-dimensional system is not always well represented by migration in a two-dimensional model $(87,99,100)$. As a result, microfluidic devices have been designed that incorporate multiple cell populations to investigate their interactions in three-dimensional environments. Interaction with endothelial cells is an important first step in neutrophil extravasation as part of their response to infection, therefore there is a particular interest in investigating neutrophilendothelial cell interactions and many microfluidic devices have been designed to study how this interaction directs the neutrophil response (101-105). A consistent theme in the design of these devices is the creation of an endothelial cell layer on a gel surface, separating neutrophils from a chemoattractant chamber (Figure 2C). These models generally use a synthetic hydrogel or collagen as the surface for growing the endothelial monolayer. Neutrophil migration across this endothelial layer is then monitored using time-lapse 
microscopy and evaluated using end point analyses. The increased physiological relevance for investigating the neutrophil response in three-dimensions and the additional imaging mechanisms available for real-time study represent significant improvements over the Transwell system, however, these devices do not include relevant architectures and additional cell types.

Recent work has demonstrated the importance of incorporating relevant three-dimensional structure into microfluidic devices for studying the innate immune system. Specifically in the case of the endothelium, it was discovered that endothelial cells have different protein expression and secretion profiles when grown in a relevant lumen geometry compared to a two-dimensional monolayer (106). This altered endothelial cell signaling could have a direct effect on neutrophil migration and function. Several microfluidic models have been developed for investigating the vasculature (107-110) and recently these models have been expanded to look at migration of neutrophils out of the vasculature. Models of the vasculature for studying signaling of the endothelium in three-dimensional architectures for the purposes of angiogenesis or inflammatory environments have been reviewed elsewhere $(111,112)$.

As the importance of physiologically relevant architectures becomes more evident, more devices are being created that incorporate geometries that mimic the in vivo environment. The most relevant architecture to be considered when studying the innate immune response to infection is the blood vessel lumen; therefore, devices that incorporate model lumens are becoming increasingly more common (Figure 2D). In general, these devices are formed by creating a hollow structure in a hydrogel that is then seeded with endothelial cells to form a luminal monolayer. The specific strategies used for fabrication, activation, and incorporation of flow into these devices differ between model designs. An early high-throughput device design used viscous finger patterning to create continuous lumens in hydrogels within microchannels (113). This approach was further developed into the LumeNEXT system (114). LumeNEXT creates lumens by polymerizing an extracellular matrix (ECM) solution around a PDMS rod that when removed leaves behind a hollow lumen. This device has been used for studies investigating the role of live pathogens (115) and cell-cell interactions, including with endothelial cells $(116,117)$, during the neutrophil inflammatory response. The LumeNEXT system has also been used in combination with the Stacks open microfluidic system (118). The Stacks system uses hollow discs filled with ECM gels stacked atop one another to create a continuous matrix. This device, and others like it, allow for the formation of soluble gradients within the matrix. Cells migrate from the lumen to a chemoattractant source through these Stacks, which are then separated allowing distinct neutrophil subpopulations to be collected based on migratory ability (119). Others have used alternative methods for fabricating lumen structures. Endothelial-lined channels have been created using in vivo images of vascular networks as a pattern to create channels using polydimethylsiloxane (PDMS). These channels contain conduits to a tissue compartment, allowing neutrophils to migrate out of the endothelium (95). Introducing a model lumen into microfluidic devices for studying innate immunity has led to new insights and opened new research directions.

The field of tissue engineering has been investigating the vascularization of hydrogels for years and has developed innovative technologies for creating vascular networks. Systems using sacrificial polymer fibers or rods, similar to the LumeNEXT device are common $(120,121)$; however, more creative approaches have also been developed. Grigoryan et al. recently developed a particularly novel method for creating a complex, entangled vascular network using food dye additives as photoabsorbers for projection soft lithography. They then used this technology to create perfusable vascular networks that model lung alveolae (122). While these technologies have not extended to the investigation of the immune system, the potential for further development of microfluidic devices mimicking in vivo biology for the investigation of the neutrophil response could use these platforms for inspiration.

A majority of the previously discussed microfluidic devices require a neutrophil isolation step in which neutrophils removed from whole blood. This isolation allows for the investigation of neutrophils specific role in innate immunity; however, it also removes neutrophils from blood components that may influence neutrophil behavior and created a neutrophil environment that is not entirely representative of in vivo conditions. Additionally, the process of neutrophil isolation from whole blood can result in artificial activation of neutrophils, leading to inaccurate results. Therefore, researchers have developed microfluidic models that allow for the use of whole blood as the source of neutrophils, eliminating the neutrophil isolation step. By circumventing the isolation step these models avoid unintentional neutrophil activation and have higher throughput than models that require neutrophil purification from blood. In general, these devices contain a whole blood loading chamber connected by a channel to a chemotaxis chamber where chemoattractant is added and neutrophil migration can be evaluated $(88,123-$ 127). Continued development of these devices will allow for further insights into signals controlling neutrophil function.

Microfluidic devices, especially those designed to use whole blood, have shown great potential for use in a variety of clinical applications. Devices allowing for the use of whole blood are particularly beneficial in clinical settings where their efficiency can have real time health implications. Additionally, these devices have a lower blood volume requirement than other device designs making them far less invasive. One such device, developed for the clinical diagnosis of asthma, isolates neutrophils from whole blood added to the system using a two-step process in which neutrophils bind to P-selectin then other blood cells are washed away. Asthma is then diagnosed using chemotaxis measurements (128). Devices have also been designed for the diagnosis of sepsis by measuring spontaneous neutrophil motility in blood $(129,130)$ and for monitoring NET prevalence within the blood post burn injury or sepsis (93). These types of whole blood microfluidic devices will continue to allow for quick and non-invasive diagnosis of a variety of innate immune related diseases. 


\section{NEUTROPHIL INTERACTIONS WITH THE BLOOD VESSEL}

During an immune response, neutrophils must first extravasate through the blood vessel before navigating the extracellular matrix to reach the site of inflammation (Figure 1). This is a multi-step process governed by interactions between neutrophils and blood vessel cells, including endothelial cells, pericytes, smooth muscle cells, and fibroblasts. Endothelial cells line the lumen of the blood vessel and are the first cells neutrophils encounter during the inflammatory response; therefore, neutrophil-endothelial cell interactions have been studied most thoroughly. Less well studied are the interactions with cells lining the sub-luminal components of the blood vessel, such as pericytes, smooth muscle cells, and fibroblasts (Table 1).

\section{Neutrophil-Endothelial Cell Interactions}

Early approaches to studying neutrophil-endothelial cell interactions employed Transwell assays and were instrumental in identifying key molecules that govern neutrophil-endothelial cell interactions during neutrophil transendothelial migration (TEM). They employed a variety of neutrophil chemoattractants produced by host cells, including IL-8, complement component $5 \mathrm{a}$ (C5a), and granulocyte-macrophage colony-stimulating factor (GM-CSF), as well as by pathogens, including fMLP and lipopolysaccharide (LPS) (65-77). These studies identified cytosolic and extracellular molecules that facilitate neutrophil TEM, including human neutrophil elastase (HNE) (70), endothelial Rho and Rho kinase (71), plasminogen activator inhibitor-1 (73), and ribosomal p70 S6 kinase (76). Other studies focused on neutrophil and endothelial cell surface proteins, such as macrophage-1 (Mac-1) and lymphocyte function-associated antigen-1 (LFA-1) on neutrophils and intercellular adhesion molecule-1 (ICAM-1), ICAM-2, and platelet-endothelial cell adhesion molecule-1 (PECAM-1) on endothelial cells, and their roles in neutrophil-endothelial cell interactions $(65,67,69,72,75)$. Furthermore, a handful of experiments have used Transwell assays to study how viral infections influence neutrophil TEM (151-153). These studies found that Respiratory Syncytial Virus-infected epithelial cells and Cytomegalovirus-infected endothelial cells induce rapid neutrophil TEM (151, 152). Studies employing Transwells have identified important signaling pathways regulating neutrophilendothelial cell interactions but only allow for end-point analysis and miss key aspects of the infectious environment, including variable presentation of soluble signals and mechanical signals.

To further elucidate factors affecting neutrophil-endothelial cell interactions, researchers have created more biologically accurate systems by designing in vitro devices that incorporate various characteristics of the in vivo environment not captured by Transwells. One such characteristic is the varying abluminal matrix stiffness found in differing tissues or disease states. A model using endothelial monolayers seeded on polyacrylamide hydrogels of varying stiffness showed neutrophil TEM increases on stiffer substrates $(131,132)$. This was accomplished by enhancing endothelial cell contractility through a myosin light chain-dependent pathway. Furthermore, this device highlighted the importance of myosin II mediated contractility and actin polymerization in neutrophils, for the speed and completion of TEM, respectively (133). Together these studies show that the physical environment affects individual cell types and subsequently alters their interactions with other cells during inflammation.

While this polyacrylamide model gives a tailorable substrate stiffness, it is restricted to chemokinetic (random migration) studies, not allowing for chemotactic (directed migration) responses. Furthermore, it does not allow for migration analysis of neutrophils in three-dimensional environments. This is significant as neutrophil integrin regulatory proteins differentially affect migration in two- and three-dimensional environments (87). Therefore, three-dimensional models allowing for analysis of neutrophil chemotaxis have been developed (Figure 2C) (101, $102,105)$. Using these devices, researchers have been able to elucidate the relative potency of various chemoattractants. It was demonstrated that AMLP is a more potent chemoattractant than IL-8 during the early stages of neutrophil TEM (101). In agreement with these results, it was determined that, in the presence of competing chemoattractant gradients, neutrophils preferentially migrate towards fMLP over IL-8 (105). Interestingly, studies with that same device found no preferential neutrophil migration between competing gradients of $\mathrm{LTB}_{4}$ and fMLP. Together, these results suggest a hierarchy in pro-migratory signals in directing neutrophil migration.

The effect of an endothelium on the neutrophil response to infections in unique organ environments, such as the lung, has been studied using microfluidic models specifically designed to replicate the in vivo environment $(103,104)$. These models stack an air channel on top of a liquid channel with a porous membrane separating the two. The membrane is seeded with epithelial and endothelial cell monolayers on the top and bottom sides, respectively. Using these lung models, researchers have found that stimulating, infecting, or replicating disease states in the epithelial cell layer activates the endothelial cell layer, resulting in neutrophil recruitment, activation, and TEM $(103,104)$.

These devices all contain a two-dimensional endothelial cell monolayer and, therefore, do not capture the in vivo architecture of the endothelium. This is important to note as the threedimensional geometry of the endothelium affects growth factor and cytokine secretion levels as well as phenotypic behavior of endothelial cells (106). For this reason, microfluidic devices have been developed to employ an endothelial lumen to more accurately capture the shape of blood vessels in vivo (Figure 2D) (114). One such device, LumeNEXT, was used to investigate the neutrophil response to the bacterial pathogen Pseudomonas aeruginosa. Interestingly, it was discovered that neutrophil lifetime and migration towards $P$. aeruginosa were significantly increased when an endothelium was present, compared to neutrophils migrating in the absence of an endothelium (116). These studies were extended by combining LumeNEXT with Stacks, as described above, to investigate the effect of transendothelial migration (TEM) on neutrophil function. 
TABLE 1 | Summary of Results.

\begin{tabular}{|c|c|c|c|c|}
\hline Cell Type & Model & Infectious Sources & Major Result & Reference \\
\hline \multicolumn{5}{|l|}{ Blood Vessel } \\
\hline \multirow[t]{7}{*}{ - Endothelial Cells } & - Transwell & $\begin{array}{l}\text { - IL8, C5a, GM-CSF, } \\
\text { fMLP, LPS }\end{array}$ & $\begin{array}{l}\text { - Human neutrophil elastase, Rho, Rho kinase, plasminogen activator inhbitor-1, and } \\
\text { ribosomal p70S6 kinase drive neutrophil TEM }\end{array}$ & $\begin{array}{c}(70,71,73, \\
76)\end{array}$ \\
\hline & - Transwell & & $\begin{array}{l}\text { - Mac-1 and LFA-1 on neutrophils and ICAM-1, ICAM-2, and PECAM-1 on } \\
\text { endothelial cells mediate neutrophil TEM }\end{array}$ & $\begin{array}{l}(65,67,69 \\
72,75)\end{array}$ \\
\hline & - $2 \mathrm{D}$ & $\begin{array}{l}\text { - TNF- } \alpha \text { Activated } \\
\text { ECs }\end{array}$ & $\begin{array}{l}\text { - Stiffer substrates enhance neutrophil TEM due to myosin-light chain dependent } \\
\text { increases in endothelial cell contractility }\end{array}$ & $(131-133)$ \\
\hline & - 3D & 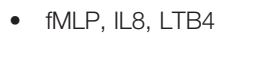 & $\begin{array}{l}\text { - } \mathrm{fMLP} \text { is a more potent neutrophil chemoattractant than IL8, suggesting a hierarchy } \\
\text { of pro-migratory signals }\end{array}$ & $\begin{array}{c}(101,102, \\
105)\end{array}$ \\
\hline & $\begin{array}{l}\text { - Organotypic } \\
\text { (LumeNEXT) }\end{array}$ & $\begin{array}{l}\text { - Bacteria } \\
\text { (P. aeruginosa) }\end{array}$ & $\begin{array}{l}\text { Endothelial secretion of IL-6 and GM-CSF enhanced neutrophil migration } \\
\text { and lifetime }\end{array}$ & $(116)$ \\
\hline & $\begin{array}{l}\text { Organotypic } \\
\text { (LumeNEXT + } \\
\text { STACKS) }\end{array}$ & - IL8 & $\begin{array}{l}\text { - TEM increases expression of genes for ROS production, cell adhesion, and } \\
\text { chemokine receptors }\end{array}$ & $(119)$ \\
\hline & $\begin{array}{l}\text { Orgoanotypic } \\
\text { (Bioinspired } \\
\text { Vasculature) }\end{array}$ & - $\quad \mathrm{fMLP}$ & $\begin{array}{l}\text { - Neutrophils adhere to endothelial cells near bifurcations in a protein kinase } \mathrm{C} \delta \text { - } \\
\text { dependent manner }\end{array}$ & $(134)$ \\
\hline \multirow[t]{2}{*}{ - Pericytes } & - Transwell & $\begin{array}{l}\text { - TNF- } \alpha \text { Activated } \\
\text { ECs/PCs }\end{array}$ & $\begin{array}{l}\text { - Endothelial cell secretion of MIF decreased pericyte contractility and barrier function } \\
\text { via reduced phospho-myosin light chain kinase }\end{array}$ & $(135-137)$ \\
\hline & - Transwell & $\begin{array}{l}\text { - IL-17 Activated } \\
\text { Pericytes }\end{array}$ & $\begin{array}{l}\text { - Conditioned media from IL-17 activated pericytes increased neutrophil polarization, } \\
\mathrm{TNF} \alpha, \mathrm{IL} 1 \alpha, \mathrm{IL} 1 \beta, \mathrm{IL} 8 \text { secretion, phagocytosis }\end{array}$ & $(81,138)$ \\
\hline - Fibroblasts & - Transwell & $\begin{array}{l}\text { - RA, UV light, cystic } \\
\text { fibrosis }\end{array}$ & - Inflammatory fibroblasts increased neutrophil adhesion to endothelial cells and TEM & $(139,140)$ \\
\hline \multicolumn{5}{|l|}{ Immune Cells } \\
\hline \multirow[t]{2}{*}{ - Neutrophils } & - $2 \mathrm{D}$ & - $\quad f M L P$ & $\begin{array}{l}\text { - At bifurcations, leading neutrophils perturb the chemoattractant gradient, directing } \\
\text { following neutrophils to take opposite path }\end{array}$ & $(141)$ \\
\hline & - Organotypic & $\begin{array}{l}\text { - Fungus } \\
\text { (A. fumigatus) }\end{array}$ & - LTB4 signaling between neutrophils leads to swarming & $\begin{array}{l}(117,142- \\
144)\end{array}$ \\
\hline - Monocytes & $\begin{array}{l}\text { - Transwell } \\
\text { - Organotypic } \\
\text { (LumeNEXT) }\end{array}$ & $\begin{array}{l}\text { - Infected monocytes } \\
\text { - A. Fungus } \\
\text { (fumigatus) }\end{array}$ & $\begin{array}{l}\text { - Neutrophils inhibit expression of IL6 and IL8 of infected monocytes } \\
\text { - Monocytes increase the neutrophil response in a MIP-1 and LPS } \\
\text { dependent manner }\end{array}$ & $\begin{array}{c}(145) \\
(117,146)\end{array}$ \\
\hline - Dendritic Cells & - Transwell & $\begin{array}{l}\text { - Fungus } \\
\text { (A. fumigatus) }\end{array}$ & $\begin{array}{l}\text { - Neutrophil derived } \alpha \text {-defensins induce DC migration and DC secretion of IL8 } \\
\text { induces neutrophil migration }\end{array}$ & $(147,148)$ \\
\hline - T Cells & - Transwell & $\begin{array}{l}\text { INF } \gamma+\text { LPS } \\
\text { Activated } \\
\text { Neutrophils }\end{array}$ & $\begin{array}{l}\text { - Activated neutrophils secrete CCL2 and CCL20, inducing Th17 chemotaxis and } \\
\text { activated Th17 cells secrete IL8, inducing neutrophil chemotaxis }\end{array}$ & $(149)$ \\
\hline \multicolumn{5}{|l|}{ Pathogens } \\
\hline \multirow[t]{3}{*}{ - Bacterium } & - Transwell & $\begin{array}{l}\text { Bacteria } \\
\text { (Escherichia coli) } \\
\text { and LPS }\end{array}$ & - Neutrophils display a more potent response to live bacteria than to LPS & $(79)$ \\
\hline & - $2 \mathrm{D}$ & - Bacteria (S. aureus) & $\begin{array}{l}\text { - Bacterial proliferation and neutrophil recruitments kinetics determine neutrophils' } \\
\text { ability to clear bacterial pathogens }\end{array}$ & (98) \\
\hline & $\begin{array}{l}\text { - Organotypic } \\
\text { (LumeNEXT) }\end{array}$ & $\begin{array}{l}\text { - Bacteria } \\
\text { (P. aeruginosa) }\end{array}$ & $\begin{array}{l}\text { - Activation of endothelial cells by } P \text {. aeruginosa led to increased neutrophil migration } \\
\text { and lifetime }\end{array}$ & (116) \\
\hline \multirow[t]{2}{*}{ - Fungus } & - $2 \mathrm{D}$ & $\begin{array}{l}\text { - Fungus } \\
\text { (A. fumigatus) }\end{array}$ & $\begin{array}{l}\text { - Chemoattractant gradients prime neutrophils to block fungal germination, leading } \\
\text { to de novo tip formation of new hyphae which is independent of NETosis and } \\
\text { NADPH oxidase activity }\end{array}$ & $(96,150)$ \\
\hline & $\begin{array}{l}\text { - Organotypic } \\
(\text { LumeNEXT) }\end{array}$ & $\begin{array}{l}\text { - Fungus } \\
\text { (A. fumigatus) }\end{array}$ & $\begin{array}{l}\text { - Neutrophils respond to } A \text {. fumigatus and their response is enhanced by paracrine } \\
\text { and autocrine signaling }\end{array}$ & $(117)$ \\
\hline
\end{tabular}

It was discovered that TEM neutrophils upregulate genes for ROS production, cell adhesion, and chemokine receptors and produce higher levels of ROS in response to phorbol-12myristate-13-acetate (PMA) stimulation compared to their non-TEM counterparts (119).
Models with relevant vascular architectures have also revealed how vessel structure alters neutrophil-endothelial cell interactions and neutrophil function. The Kiani Lab found that neutrophils preferentially adhere to activated endothelial cells near bifurcations in a protein kinase $\mathrm{C} \delta$-dependent manner 


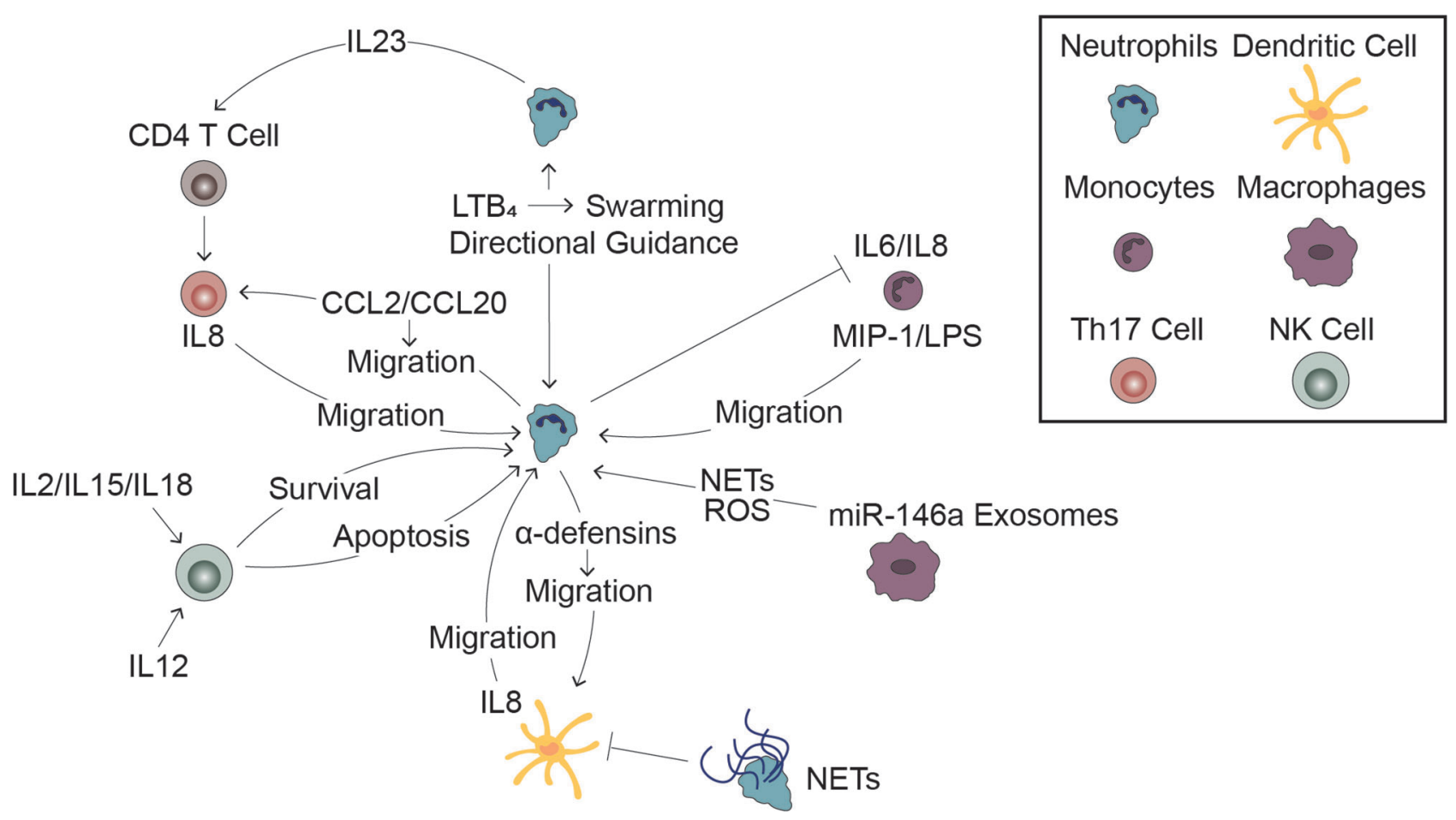

FIGURE 3 | Interactions with Immune Cells Influences the Neutrophil Response. Cell-cell signaling between neutrophils and other immune cells plays a significant role in the innate immune response to infection. Leading neutrophils influence swarming and the directional migration of trailing neutrophils (top middle). Monocytes induce neutrophil migration and in turn, neutrophils inhibit pro-inflammatory signaling by monocytes (top right). Release of miR-146a rich exosomes induces neutrophil extracellular trap formation and reactive oxygen species generation (bottom right). Dendritic cells stimulate neutrophil migration while neutrophils have a dual effect on dendritic cells, stimulating migration through release of $\alpha$-defensins while reducing DC production of inflammatory signals through signaling through NETs (bottom middle). NK cells can both promote neutrophil survival following stimulation by pro-inflammatory cytokines and promote neutrophil apoptosis following stimulation with anti-inflammatory cytokines (bottom left). Neutrophils stimulate Th17 cell migration and induce CD4 T cells to become Th17 cells, which in turn stimulate neutrophil migration (top left).

using a model vasculature, patterned to mimic an in vivo network (134). This result was particularly interesting as it was previously discovered, using a device with a tailorable bifurcations, that wider bifurcation angles facilitate increased neutrophil adhesion (154). Together, these results demonstrate the importance of vascular architecture and the consideration of both luminal structure and vascular bifurcations when studying neutrophil-endothelial cell interactions.

A wide range of in vitro models have been used to study neutrophil-endothelial cell interactions. Each system has its noted advantages and disadvantages, making them ideal for different types of studies. From identifying neutrophil chemoattractants and endothelial cell activators to elucidating the impacts of the physical environment on cell-cell interactions, these tools help us deepen our understanding of neutrophilendothelial cell interactions during the inflammatory response.

\section{Neutrophil-Pericyte Interactions}

After extravasation through the endothelium, neutrophils encounter pericytes in the basement membrane of the blood vessel. Pericytes serve to physically stabilize blood vessels, regulate blood flow, and aid in vascular development, maturation, remodeling, and permeability. These distinct pericyte roles have been previously reviewed (155). More recently, studies have investigated the role of pericytes in neutrophil extravasation and migration. The Gonzalez Lab has conducted extensive research on the effects of pericytes in neutrophil TEM using endothelial cell and pericyte monolayers in Transwell assays (80,81, 135-138). Through a series of studies, they found that tumor necrosis factor- $\alpha$ (TNF- $\alpha$ ) activation of endothelial cells and pericytes generates competing pro- and anti-inflammatory signals. Specifically, they showed neutrophils migrate through pericyte monolayers to a lesser extent than they do through endothelial cell monolayers and migrate through bilayers of the two cell types at intermediate levels (135). They then demonstrated that the observed intermediate migration is, in part, due to the disparate effects of TNF- $\alpha$ on endothelial cells and pericytes. TNF- $\alpha$ activation has a pro-inflammatory effect on endothelial cells, inducing the secretion of macrophage migration inhibitory factor (MIF), which has a pro-inflammatory effect on pericytes $(136,137)$. Conversely, TNF- $\alpha$ activation has an antiinflammatory effect on pericytes, leading to a decrease in the inflammatory phenotype of endothelial cells as indicated by 
decreased neutrophil TEM across endothelial monolayers treated with supernatants from TNF- $\alpha$ treated pericytes (136). Furthermore, they found that seeding endothelial monolayers on pericyte-derived basement membrane decreased neutrophil adhesion and migration through the endothelium. Specifically, they found inflammatory stimuli led to fibronectin-rich, collagen-poor protein deposition as well as augmented fibronectin and laminin specific MMP production. Both these factors facilitated increased neutrophil migration (81, 138). Together, these results imply that endothelial cell-pericyte paracrine signaling is important in the regulation of neutrophil transmigration. They also suggest in vitro models that do not incorporate pericytes may miss relevant interactions affecting neutrophil migration.

While these studies demonstrated the importance of pericytes in neutrophil migration, they were carried out using Transwell assays which do not allow for real-time investigation of neutrophil interactions with pericytes, and more complex in vitro devices have not been used to investigate the intricacies of human neutrophil-pericyte interactions in relevant architectures. These studies indicate that endothelial-pericyte interactions likely play an important role in neutrophil TEM. Endothelial cell signaling is highly dependent on physical cues such as flow and monolayer architecture; therefore, important signaling pathways may not be captured in simple Transwell models. The interaction of pericytes with endothelial cells in a lumen geometry has been investigated in the context of tissue engineering. Alimperti et al. developed a bicellular device including endothelial lumens surrounded by attached pericytes, or human bone marrow stromal cells exhibiting mural cell characteristics similar to pericytes (156). They demonstrated that while RhoA activity increases vascular permeability, Rac1 and $\mathrm{N}$-cadherin help maintain barrier function. Additionally, they found LPS, TNF- $\alpha$, and thrombin increased vascular permeability and resulted in the detachment of the stromal cells from the endothelial lumen. The detachment of pericytes from the endothelium during inflammation is not captured by Transwell studies. As such, physiologically relevant in vitro models are needed to study the role of this phenomenon during inflammation. In the future, this model could be adapted to study the effect of pericytes on neutrophil migration in a relevant in vitro environment.

\section{Neutrophil-Smooth Muscle Cell Interactions}

After extravasation through the basement membrane, neutrophils must navigate their way through smooth muscle cells. It has been shown that exosomes and nitric oxide, secreted by neutrophils in response to bacterial stimulators (LPS or fMLP), alter smooth muscle cell morphology and functionality $(157,158)$. Furthermore, it is known that smooth muscle cells secrete IL-8 in response to IL-17 stimulation (159). Despite evidence of neutrophil-smooth muscle cell interactions during inflammation, sophisticated in vitro devices emulating this part of the in vivo environment have yet to be developed to study the crosstalk between these cells. However, a lung airway-on-a-chip model for studying interactions between the epithelium and smooth muscle cells has been previously created (160). The design of this device could be used to generate a similar onchip platform for studying neutrophil-smooth muscle cell interactions during inflammation.

\section{Neutrophil-Fibroblast Interactions}

Fibroblasts are present in the outer most layer of blood vessels, the adventitia. Fibroblasts are the main producers of ECM proteins and thus are crucial in tissue regeneration (161). However, they also play significant roles in inflammatory responses, as reviewed previously $(162,163)$. Early studies used simple culturing techniques to demonstrate fibroblasts' ability to produce IL-8 (164). These culturing studies then advanced to separate neutrophils and fibroblasts with a Transwell filter coated with endothelial cells (165). These studies showed synovial fibroblasts from patients with rheumatoid arthritis increased the number of neutrophils adhering to endothelial cells compared to their healthy counterparts. Recently, a device incorporating a flat endothelium, fibroblasts in a sub-luminal collagen gel, and a keratinocyte monolayer was developed (139). Using this model, Kwak et al. demonstrated ultraviolet lightinduced cytokine secretion by the resident cells increased neutrophil TEM. Mejías et al. developed a system for studying neutrophil recruitment from the vascular network incorporating flat epithelial and endothelial monolayers in addition to fibroblasts (140). By replacing the healthy epithelium with cystic fibrosis human bronchial epitheliums, they were able to induce a disease state that resulted in neutrophil recruitment. These sophisticated devices should serve as models for introducing additional cell types and reconfiguring the geometries to make even more physiologically relevant systems in the future.

Neutrophil-endothelial cell interactions have been studied thoroughly, in both simple and complex in vitro systems. However, evidence suggests that other vascular and stromal cells, including pericytes, smooth muscle cells, and fibroblasts impact neutrophil inflammatory responses. While some microfluidic devices have been created to incorporate subsets of these cells in one system, future work is needed to make more biologically accurate replications of blood vessels and the surrounding tissue that contain all relevant cells populations.

\section{NEUTROPHIL INTERACTIONS WITH IMMUNE CELLS}

Inside of blood vessels, neutrophils communicate with other immune cells to efficiently navigate the vascular network and rapidly activate during an inflammatory response. Once outside of the blood vessel, neutrophils must maneuver through the ECM to reach the site of inflammation. Neutrophils achieve this by following various chemoattractant gradients generated by both pathogens and other host cells, including other immune cells (Figure 1). Furthermore, immune cells help activate and 
inactivate neutrophils as is necessary for inducing, sustaining, and eventually terminating inflammation (Figure 3).

\section{Neutrophil-Neutrophil Interactions}

Neutrophils signal to other neutrophils during inflammatory responses, generally through a signal amplification mechanism. They are the primary source of $\mathrm{LTB}_{4}$, a proinflammatory lipid mediator, which is critical for the neutrophil response to bacterial, fungal, and parasitic infections $(38,117,166)$. At sites of infection, neutrophil production of $\mathrm{LTB}_{4}$ has been shown to be important for swarming, a phenomenon by which neutrophils surround a pathogen and encase it to prevent it from spreading throughout the body $(97,167)$. Interestingly, the swarming response orchestrated by $\mathrm{LTB}_{4}$ varies in magnitude depending on the pathogen type, indicating a role for the pathogen as well in modulating this neutrophil function (97). While neutrophil swarming has primarily been studied in vivo, physiologically relevant microfluidic models of the neutrophil response have also shown $\mathrm{LTB}_{4}$-mediated neutrophil swarming in response to $A$. fumigatus (117, 142-144).

In addition to amplifying chemotactic signals through secondary gradients of $\mathrm{LTB}_{4}$, neutrophils communicate inside blood vessels by altering the physical environment. The vasculature is a complex network, and neutrophils must be able to navigate this network without forming "traffic jams." To investigate how neutrophils avoid build ups, Wang et al. developed a field-goal shaped device where the channels create a " $T$ " then continue up from both branches, simulating a vascular bifurcation (141). By controlling the width of the branch channels and the distance between neutrophils approaching the decision point, they determined leading neutrophils perturb the chemoattractant gradient and, to a lesser extent, the pressure gradient in the branch they traverse, causing closely trailing neutrophils to enter the opposite branch. Whether inside a blood vessel prior to activation or in the extracellular matrix during an inflammatory response, neutrophils are constantly communicating with one another to ensure they can respond rapidly and efficiently when the innate immune response is initiated.

\section{Neutrophil-Monocyte/Macrophage/ Dendritic Cell Interactions}

In addition to neutrophils, monocytes extravasate through the blood vessel and into the surrounding tissue following an inflammatory insult. Once in the tissue, they differentiate into either macrophages or dendritic cells, in response to the surrounding environmental cues $(168,169)$. Monocytes in each of these differentiation states have been shown to interact with neutrophils. Through a co-culturing method where monocytes and neutrophils were either allowed to have direct contact with one another or were separated by a Transwell filter, Tang et al. demonstrated neutrophils inhibit the expression of IL- 6 and IL- 8 by rhinovirus infected monocytes, reducing inflammatory signal production (145). Furthermore, using the LumeNEXT device, it was discovered that monocytes increase the neutrophil migratory response to $A$. fumigatus infection through a mechanism involving MIP-1 and to LPS in an extracellular nucleotidedependent manner $(117,146)$.

Complex, three-dimensional microfluidic devices have yet to be designed to study neutrophil-macrophage interactions. However, simple in vitro approaches have been used to study the crosstalk between these cell types. It has also been shown oxidized low-density lipoproteins induce secretion of miR-146a rich exosomes by macrophages, which generate oxidative stress in neutrophils, increasing neutrophil ROS production and NET formation (170). Additionally, in a co-culturing study, neutrophils and macrophages were shown to cooperate in a contact dependent manner to eliminate macrophages infected with Leishmania braziliensis (171).

Like neutrophil-macrophage interactions, neutrophildendritic cell interactions have not been studied in complex devices mimicking the in vivo environment, but simple in vitro studies have been conducted. Transwell assays have been used to show $\alpha$-defensins, proinflammatory peptides derived from neutrophils, induce immature dendritic cell migration and $A$. fumigatus-infected dendritic cells induce neutrophil migration through secretion of IL-8 $(147,148)$. Furthermore, incubating dendritic cells with NETs prior to LPS exposure was found to attenuate upregulation of dendritic cell markers and secretion of inflammatory cytokines (172).

Dendritic cells and macrophages play a crucial role in the both the innate and adaptive immune responses, but we have only begun to understand how interactions with these cells guide the neutrophil response. Current studies primarily use simple Transwell assays that do not allow for a detailed evaluation of neutrophil activation or function following interaction with monocytic cells; therefore, these studies should be expanded to complex devices that allow for real-time analysis.

\section{Neutrophil-Platelet Interactions}

Platelets are non-nucleated, membrane bound packets of cytoplasm that are released into the blood by megakaryocytes residing in the bone marrow. These cell fragments predominantly serve to maintain blood vessel integrity as well as initiate and participate in clotting. Prior to extravasation, neutrophils interact with platelets in the blood. Most work investigating neutrophil-platelet interactions has focused on their interplay during thrombosis. Transwell studies showed that platelet factor 4 , which is secreted by activated platelets, serves as a chemoattractant for neutrophils (173). Once drawn to the platelets, neutrophils adhere to platelets through selectinligand binding ( $\mathrm{P}$-selectin on platelets and L-selectin on neutrophils), as was demonstrated in parallel plate flow chambers (174). Flow chambers have also been used to show that platelets indirectly affect neutrophils by releasing plateletderived extracellular vesicles (PEVs) that transiently bind to either endothelial cells or neutrophils. Upon binding PEVs induced increased adhesion molecule (ICAM-1 and VCAM-1) expression by endothelial cells and integrin (CD11b) expression on neutrophils, which promotes increased neutrophilendothelial cell adhesion interactions (175). Collectively, these results indicate that platelets play a regulatory role in neutrophil 
transendothelial migration, yet their contribution to the neutrophil response to infection has not been studied in depth. Further investigation into the role of platelets in the neutrophil response should be conducted in multicellular systems that include endothelial cells, better recapitulating the in vivo environment.

\section{Neutrophil-Other Immune Cell Interactions}

Of the remaining neutrophil-immune cell interactions, most in vitro work has been conducted with Transwell assays or by coculture of cells with and without porous membrane dividers. Neutrophil interactions with T cells, mast cells, and natural killer cells (NK cells) have been studied using these approaches. Neutrophil-T cell Transwell assays have revealed neutrophils induce Th17 chemotaxis by secreting CCL2 and CCL20 and activated Th17 cells secreted IL- 8 for the recruitment of neutrophils (149). Furthermore, Toll-like Receptor 8 (TLR8) activated neutrophils have been demonstrated to secrete IL-23, which induces naïve CD4 T cells to become Th17 cells (176). Numerous mast cell studies have shown stimulation with various pathogens or pathogen-derived molecules induces secretion of inflammatory cytokines and chemokines that augment neutrophil migration $(28,177-179)$. Lastly, neutrophil-NK cell studies have revealed both contact dependent and independent crosstalk occur between the cells leading to changes in neutrophil receptor expression and survival. NK cells stimulated with proinflammatory cytokines, including IL-2, IL-15, and IL-18, send neutrophil survival signals, and increase expression of Fc $\gamma$ receptor I (CD64), CD11b, and CD69 on neutrophils (180). Conversely, NK cells can also induce apoptosis of neutrophils in a caspase and direct contact dependent manner in response to IL-12, an anti-inflammatory cytokine (181). NK cells can also bring about neutrophil apoptosis following ROS production through binding of the MIC-A protein on neutrophils with the NKG2D protein on NK cells (182).

Neutrophils signal to and receive signals from numerous immune cell types that guide and regulate their activity during an inflammatory response. This cellular crosstalk has been investigated primarily in animal models and simple in vitro studies, primarily co-cultures of neutrophils with another cell type. In the same way Transwell inserts were used to discover key signaling molecules and interactions between neutrophils and endothelial cells or pericytes, co-culture studies have identified molecules immune cells use to signal to neutrophils. However, more complex microfluidic devices are needed to capture the nuances of these cell-cell interactions in more physiologically relevant environments.

\section{NEUTROPHIL INTERACTIONS WITH PATHOGENS}

Cellular signaling between a pathogen source and responding neutrophils plays a key and obvious role in directing the overall innate immune response. Pathogens release several signals driving the neutrophil response. Initially, pathogen derived peptides activate endothelial cells lining the blood vessel initiating the neutrophil extravasation process described previously. Once neutrophils have left the blood vessel, they reach the site of infection by following PAMPs and pathogen derived peptides released by the pathogen source (Figure 1). Recent work has shown the neutrophil response is dependent on the specific pathogen causing the inflammation (79). This suggests the innate immune response is tuned to respond specifically to distinct bacteria or fungi infecting the body. In responding to a pathogen source, neutrophils have multiple tools in their arsenal to use in attacking and slowing down the spread of the infection, including phagocytosis, swarming, ROS generation, and NETosis. Just as cellular interactions direct the migratory response of neutrophils towards the pathogen source, they also modulate the use of these antimicrobial response tools. Microfluidic devices have been created to investigate the specific and unique role the pathogen source plays in both directing neutrophil migration and modulating neutrophil effector functions upon interaction with the pathogen itself.

\section{Neutrophil-Bacterium Interactions}

Neutrophil-pathogen interactions have been investigated since neutrophils were first discovered in the $19^{\text {th }}$ century. Early modern studies investigated the interactions between bacteria and neutrophils by introducing both cell types into solution together and monitoring the results (183). These studies primarily focused on the metabolic pathways involved during the interaction and the effects on respiration. As the field has evolved, the research focus has shifted to investigating the specific signaling events between the pathogen and neutrophils. The methods used for investigating these interactions have developed with improved experimental models. An early model design used a simple Transwell assay where neutrophils were seeded in the upper chamber and live, intact bacteria were seeded in the lower chamber to investigate if different pathogens distinctly directed the neutrophil response to infection (79). Significantly, this paper demonstrated neutrophils display a more potent response to the live, intact bacteria than to isolated bacterial peptides. Specifically, they found neutrophil migration towards Escherichia coli occurred at a rate ten-fold greater than towards LPS. While many current and past studies solely use bacterial peptides as their model for infection (66-77), this paper highlights the importance of using whole bacteria. Microfluidic devices of increasing physiological relevance have been designed to further investigate neutrophil-pathogen interactions. One such device used 'war theatres' (Figure 2B, bottom) to identify that bacterial proliferation and neutrophil recruitment kinetics were important factors in determining pathogen infection outcomes, as measured by neutrophils' ability to neutralize bacteria (98). Advanced microfluidic devices incorporating physiologically relevant architectures and additional cellular components have also been designed to investigate neutrophil-pathogen interactions. It was discovered using an organotypic lumen model that activation of an endothelial lumen by the bacterial pathogen Pseudomonas aeruginosa led to an enhanced neutrophil response due to increased endothelial secretion of IL- 6 and GM-CSF (116). This paper investigated neutrophil recruitment to a 
single bacterial source, but additional research is needed to further understand migration towards other types of bacterial pathogens.

\section{Neutrophil-Fungus Interactions}

In addition to studying interactions between bacteria and neutrophils, several groups have investigated interactions between fungi and neutrophils, with many studies focusing on the environmental fungus A. fumigatus. A. fumigatus is a common opportunistic pathogen that, while harmless to most people, can cause life-threatening disease in immunocompromised individuals (184). It is well-studied, making it a good model pathogen for studying innate immunity in microfluidic devices. A series of studies using devices specifically designed for examining neutrophil interactions with $A$. fumigatus show neutrophils limit $A$. fumigatus growth through a variety of mechanisms. Jones et al. found an introduced chemoattractant gradient primed neutrophils to migrate to A. fumigatus conidia and block fungal germination and growth (96). Interestingly, this blockage in hyphal growth is counteracted by the fungus via de novo tip formation and development of a new hyphae near the interaction site. This fungal behavior was discovered and found to be independent of both NADPH oxidase activity and NETosis using a microfluidic device allowing for single cell analysis of fungal-neutrophil interactions (Figure 2B, bottom) (150). In the presence of high numbers of neutrophils, this leaves the hyphae vulnerable, but without a significant neutrophil response it can lead to aggressive $A$. fumigatus invasion, potentially describing a mechanism by which neutrophils protect against invasive $A$. fumigatus infections. Finally, studies have used unique microfluidic platforms to investigate molecules with therapeutic potential. One such study describes bifunctional compounds that bind to both the microbial target $(A$. fumigatus) and the neutrophil chemoattractant receptors. These compounds were able to assist neutrophils in slowing hyphal growth and were also able to enhance phagocytosis of conidia (185).

Neutrophil interactions with fungal infections have also been investigated in three-dimensional models containing more physiologically relevant components and architectures. The ability of neutrophils to migrate in three dimensions in these models is an improvement over previous two-dimensional devices as migration in a three-dimensional environment is not always well represented by migration in a two-dimensional model $(87,99,100)$ Importantly, these models include endothelial cells which play an important role in modulating the innate immune response. In vivo studies have demonstrated a role for endothelial cells in altering the activation state of neutrophils and altering their response to infection (186). Therefore, it is critical to include these physiologically relevant architectures and components to elucidate an accurate understanding of neutrophil-pathogen interactions. Several three-dimensional microfluidic devices have been created with thematically similar designs but slight variations between them. In general, these devices consist of an endothelial cell coated lumen with neutrophils seeded within and a fungal source outside (Figure 2D) (115). Studies using three-dimensional models have found paracrine signaling, from monocytes through MIP-1 and other neutrophils through $\mathrm{LTB}_{4}$, plays an important role in driving the neutrophil response towards a source of A. fumigatus (117). These devices have provided new insights into the neutrophil response to fungal infections and identified paracrine signaling mechanisms influencing this response; however, in vivo studies point to an important role for other cell types, including macrophages, in regulating the neutrophil response to fungal infections. New devices containing additional relevant cell types are needed to fully understand the neutrophil response to infection.

Not only have microfluidic devices been designed to investigate interactions between neutrophils and different pathogen types; they have also been designed for investigating how neutrophils use different effector functions to combat these pathogens. In general, these devices involve neutrophils being captured, either by P-selectin (92) or by micropost arrays (93), and stained to visualize NET formation and ROS production under different conditions. One result of interest showed ROS production was necessary for neutrophils to form NETs (92). Studies using these devices have also shown an increase in NETs circulating in the blood following a major burn (93). Neutrophil use of effector functions is tightly regulated by cellcell interactions.

Pathogens play an important signaling role in modulating neutrophil migration and effector functions as demonstrated by the papers discussed above. A common theme among papers investigating neutrophil-pathogen interactions is the diversity of neutrophil responses arising from different pathogens. This points to a critical need to better understand the uniqueness in each neutrophil-pathogen interaction and what drives these differences. The devices highlighted in this section have already played an important role in expanding the depth of understanding of this important relationship, but more work must be done to further our understanding of how cell-cell interactions contribute to the complicated signaling networks driving the neutrophil response. Specifically, the interaction of neutrophils with other tissue cells must be included in the analysis of neutrophils' response to pathogens. It is known that interaction with vascular and other immune cells alters the activation state of neutrophils in vitro (116) and in vivo (85, 187); therefore, analysis of neutrophil interactions with pathogens in systems that do not include supporting cell populations give an incomplete picture of neutrophil activation and function.

\section{CONCLUSION}

A properly regulated neutrophil response is critical for fighting infection while maintaining tissue homeostasis. Following infection, neutrophils must process a complex milieu of signals emanating from the cellular and physical components of their environment into an efficient and directed response. There has been a substantial effort to understand how the various signals neutrophils encounter drive their response using both in vivo 
and in vitro models. Numerous signaling pathways and cell-cell interactions have been identified as critical regulators of the neutrophil response using these models, but we still do not have a clear picture of how these signals are integrated into a single response following an infection. Our lack of understanding is derived from a few key limitations of current experimental systems. Animal models provide a complete overview of an immune response but do not always correlate to human disease and most current in vitro models lack at least one key component of an infectious microenvironment: a live source of infecting pathogen, relevant cell populations, and relevant architectures. To create a full picture of the signaling networks driving the neutrophil response, we must strive to develop new models, inspired by in vivo biology, that capture all relevant aspects of the infectious microenvironment. Initially, studies must be conducted to increase our understanding of how interactions with different cell types (smooth muscle cells, fibroblasts, macrophages, dendritic cells, NK and T cells) alter neutrophil function and activation using simple in vitro devices that allow for real-time analysis. To date, these studies have primarily been conducted using Transwell assays which prevent investigation into the morphologies of interacting cells, the modes of interaction, and the kinetics of neutrophil activation and function. These studies will incrementally increase our understanding of neutrophil function in the infectious microenvironment. Future studies should then focus on the development of devices that can include multiple cell populations known to influence the neutrophil response to

\section{REFERENCES}

1. Wright HL, Moots RJ, Edwards SW. The Multifactorial Role of Neutrophils in Rheumatoid Arthritis. Nat Rev Rheumatol (2014) 10:593-601. doi: 10.1038/nrrheum.2014.80

2. Mitroulis I, Kambas K, Ritis K. Neutrophils, IL-1 $\beta$, and Gout: Is There a Link? Semin Immunopathol (2013) 35:501-12. doi: 10.1007/s00281-0130361-0

3. Boxer L, Dale DC. Neutropenia: Causes and Consequences. Semin Hematol (2002) 39:75-81. doi: 10.1053/shem.2002.31911

4. Johnston RB. Clinical Aspects of Chronic Granulomatous Disease. Curr Opin Hematol (2001) 8:17-22. doi: 10.1097/00062752-200101000-00004

5. Etzioni A, Frydman M, Pollack S, Avidor I, Phillips ML, Paulson JC, et al. Recurrent Severe Infections Caused by a Novel Leukocyte Adhesion Deficiency. N Engl J Med (1992) 327:1789-92. doi: 10.1056/ NEJM199212173272505

6. Filippi MD. Neutrophil Transendothelial Migration: Updates and New Perspectives. Blood (2019) 133:2149-58. doi: 10.1182/blood-2018-12844605

7. Vestweber D. How Leukocytes Cross the Vascular Endothelium. Nat Rev Immunol (2015) 15:692-704. doi: 10.1038/nri3908

8. De Oliveira S, Rosowski EE, Huttenlocher A. Neutrophil Migration in Infection and Wound Repair: Going Forward in Reverse. Nat Rev Immunol (2016) 16:378-91. doi: 10.1038/nri.2016.49

9. Kruger P, Saffarzadeh M, Weber ANR, Rieber N, Radsak M, von Bernuth H, et al. Neutrophils: Between Host Defence, Immune Modulation, and Tissue Injury. PLoS Pathog (2015) 11:1-23. doi: 10.1371/journal.ppat.1004651

10. Zindel J, Kubes P. DAMPs, PAMPs, and LAMPs in Immunity and Sterile Inflammation. Annu Rev Pathol Mech Dis (2020) 15:493-518. doi: 10.1146/ annurev-pathmechdis-012419-032847

11. Kanwar S, Bullard DC, Hickey MJ, Smith CW, Beaudet AL, Wolitzky BA, et al. The Association Between $\alpha 4$-Integrin, P-selectin, and E-selectin in an infection, including macrophages, dendritic cells, pericytes, and stromal cells to investigate the complex cellular crosstalk occurring in an environment that most closely recapitulates tissues in vivo. This will require significant design optimization as different cell populations require diverse culture times, nutrients, and conditions in vitro; therefore, the authors suggest an iterative process in which single cell populations are included in each new design. In the design of these devices, special attention should be paid to integrate cellular populations that are most likely involved in multicellular signaling cascades that influence neutrophil migration using results from simple in vitro studies and in vivo work as a guide. Finally, evidence suggests that live pathogens provide a more relevant stimulus than peptides, yet many studies investigating neutrophil function still rely on the introduction of inflammatory peptides or single attractants. Therefore, there should be a significant emphasis in the field to use a variety of live pathogens to simulate infections rather than relying on bacterial derived peptides. By understanding how cell-cell interactions regulate the neutrophil response to infection, we can attempt to manipulate the response through the intelligent development of new therapeutics to treat infection.

\section{AUTHOR CONTRIBUTIONS}

IR, CC, and LH wrote and edited the manuscript. All authors contributed to the article and approved the submitted version.

Allergic Model of Inflammation. J Exp Med (1997) 185:1077-87. doi: 10.1084/jem.185.6.1077

12. Mcever RP. Selectins: Initiators of Leucocyte Adhesion and Signalling At the Vascular Wall. Cardiovasc Res (2015) 107:331-9. doi: 10.1093/cvr/ cvv154

13. Phillipson M, Heit B, Colarusso P, Liu L, Ballantyne CM, Kubes P. Intraluminal Crawling of Neutrophils to Emigration Sites: A Molecularly Distinct Process From Adhesion in the Recruitment Cascade. J Exp Med (2006) 203:2569-75. doi: 10.1084/jem.20060925

14. Schenkel AR, Mamdouh Z, Muller WA. Locomotion of Monocytes on Endothelium is a Critical Step During Extravasation. Nat Immunol (2004) 5:393-400. doi: 10.1038/ni1051

15. Woodfin A, Reichel CA, Khandoga A, Corada M, Voisin MB, Scheiermann C, et al. JAM-A Mediates Neutrophil Transmigration in a Stimulus-Specific Manner In Vivo: Evidence for Sequential Roles for JAM-A and PECAM-1 in Neutrophil Transmigration. Blood (2007) 110:1848-56. doi: 10.1182/blood2006-09-047431

16. Marmon S, Hinchey J, Oh P, Cammer M, De Almeida CJ, Gunther L, et al. Caveolin-1 Expression Determines the Route of Neutrophil Extravasation Through Skin Microvasculature. Am J Pathol (2009) 174:684-92. doi: 10.2353/ajpath.2009.080091

17. Vaporciyan AA, Delisser HM, Yan Hc, Mendiguren II, Thorn SR, Jones ML, et al. Involvement of Platelet-Endothelial Cell Adhesion Molecule-1 in Neutrophil Recruitment In Vivo. Science (80- ) (1993) 262:1580-2. doi: $10.1126 /$ science. 8248808

18. Wegmann F, Petri B, Khandoga AG, Moser C, Khandoga A, Volkery S, et al. ESAM Supports Neutrophil Extravasation, Activation of Rho, and VEGFinduced Vascular Permeability. J Exp Med (2006) 203:1671-7. doi: 10.1084/ jem. 20060565

19. Heck LW, Blackburn WD, Irwin MH, Abrahamsont DR. Degradation of Basement Membrane Laminin by Human Neutrophil Elastase and Cathepsin-G. Am J Pathol (1990) 136:1267-74. 
20. Maus UA, Waelsch K, Kuziel WA, Delbeck T, Mack M, Blackwell TS, et al. Monocytes are Potent Facilitators of Alveolar Neutrophil Emigration During Lung Inflammation: Role of the CCL2-CCR2 Axis. J Immunol (2003) 170:3273-8. doi: 10.4049/jimmunol.170.6.3273

21. Futosi K, Fodor S, Mócsai A. International Immunopharmacology Reprint of Neutrophil Cell Surface Receptors and Their Intracellular Signal Transduction Pathways. Int Immunopharmacol (2013) 17:1185-97. doi: 10.1016/j.intimp.2013.11.010

22. Broggi A, Granucci F. Microbe- and Danger-Induced Inflammation. Mol Immunol (2015) 63:127-33. doi: 10.1016/j.molimm.2014.06.037

23. Vénéreau E, Ceriotti C, Bianchi ME. Damps From Cell Death to New Life. Front Immunol (2015) 6:422. doi: 10.3389/fimmu.2015.00422

24. Cordeiro J, Jacinto A. The Role of Transcription-Independent Damage Signals in the Initiation of Epithelial Wound Healing. Nat Rev Mol Cell Biol (2013) 14:249-62. doi: 10.1038/nrm3541

25. Becker S, Quay J, Soukup J. Cytokine (Tumor Necrosis Factor, IL-6, and IL8) Production by Respiratory Syncytial Virus-Infected Human Alveolar Macrophages. J Immunol (1991) 147:4307-12.

26. Sacramento L, Trevelin SC, Nascimento MS, Lima-Jùnior DS, Costa DL, Almeida RP, et al. Toll-Like Receptor 9 Signaling in Dendritic Cells Regulates Neutrophil Recruitment to Inflammatory Foci Following Leishmania Infantum Infection. Infect Immun (2015) 83:4604-16. doi: 10.1128/IAI.00975-15

27. Gong T, Liu L, Jiang W, Zhou R. DAMP-Sensing Receptors in Sterile Inflammation and Inflammatory Diseases. Nat Rev Immunol (2020) 20:95112. doi: 10.1038/s41577-019-0215-7

28. Möller A, Lippert U, Lessmann D, Kolde G, Hamann K, Welker P, et al. Human Mast Cells Produce IL-8. J Immunol (1993) 151:3261-6.

29. Smart SJ, Casale TB. TNF- $\alpha$-Induced Transendothelial Neutrophil Migration is IL-8 Dependent. Am J Physiol (1994) 266:L238-45.

30. Ebrahimzadeh PR, Hogfors C, Braide M. Neutrophil Chemotaxis in Moving Gradients of fMLP. J Leukoc Biol (2000) 67:651-61. doi: 10.1002/ jlb.67.5.651

31. Russo RC, Garcia CC, Teixeira MM, Amaral FA. The CXCL8/IL-8 Chemokine Family and its Receptors in Inflammatory Diseases. Expert Rev Clin Immunol (2014) 10:593-619. doi: 10.1586/1744666X.2014.894886

32. Miller LS, Pietras EM, Uricchio LH, Hirano K, Rao S, Lin H, et al. Inflammasome-Mediated Production of IL-1 $\beta$ Is Required for Neutrophil Recruitment Against Staphylococcus Aureus In Vivo. J Immunol (2007) 179:6933-42. doi: 10.4049/jimmunol.179.10.6933

33. Tiku K, Tiku ML, Skosey JL. Interleukin-1 Production by Human Polymorphonuclear Neutrophils. J Immunol (1986) 136:3677-85.

34. Dinarello CA. Overview of the IL-1 Family in Innate Inflammation and Acquired Immunity. Immunol Rev (2018) 281:8-27. doi: 10.1111/imr.12621

35. Martin TR, Altman LC, Albert RK, Henderson WR. Leukotriene B4 Production by Human Alveolar Macrophage: A Potential Mechanism for Amplifying Inflammation in the Lung. Am Rev Respir Dis (1984) 129:106-11.

36. Fels AOS, Pawlowski NA, Cramer EB, King TK, Cohn ZA, Scott WA. Human Alveolar Macrophages Produce Leukotriene B4. Proc Natl Acad Sci USA (1982) 79:7866-70. doi: 10.1073/pnas.79.24.7866

37. Ribeiro RA, Souza-Filho MVP, Souza MHLP, Oliveira SHP, Costa CHS, Cunha FQ, et al. Role of Resident Mast Cells and Macrophages in the Neutrophil Migration Induced by LTB4, fMLP, and C5a Des Arg. Int Arch Allergy Immunol (1997) 112:27-35. doi: 10.1159/000237427

38. Doerfler ME, Danner RL, Shelhamer JH, Parillo JE. Bacterial Lipopolysaccharides Prime Human Neutrophils for Enhanced Production of Leukotriene B4. J Clin Invest (1989) 83:970-7. doi: 10.1172/JCI113983

39. Canetti C, Silva JS, Ferreira SH, Cunha FQ. Tumour Necrosis Factor-Alpha and Leukotriene B4 Mediate the Neutrophil Migration in Immune Inflammation. Br J Pharmacol (2001) 134:1619-28. doi: 10.1038/sj.bjp. 0704403

40. Chen M, Lam BK, Kanaoka Y, Nigrovic PA, Audoly LP, Austen KF, et al. Neutrophil-Derived Leukotriene B4 is Required for Inflammatory Arthritis. J Exp Med (2006) 203:837-42. doi: 10.1084/jem.20052371

41. Lämmermann T. In the Eye of the Neutrophil Swarm-Navigation Signals That Bring Neutrophils Together in Inflamed and Infected Tissues. J Leukoc Biol (2016) 100:55-63. doi: 10.1189/jlb.1mr0915-403
42. Lämmermann T, Afonso P, Angermann BR, Wang JM, Kastenmüller W, Parent CA, et al. Neutrophil Swarms Require LTB4 and Integrins At Sites of Cell Death In Vivo. Nature (2013) 498:371-5. doi: 10.1038/nature12175

43. Nordenfelt P, Tapper H. Phagosome Dynamics During Phagocytosis by Neutrophils. J Leukoc Biol (2011) 90:271-84. doi: 10.1189/jlb.0810457

44. Lee WL, Harrison RE, Grinstein S. Phagocytosis by Neutrophils. Microbes Infect (2003) 5:1299-306. doi: 10.1016/j.micinf.2003.09.014

45. Dahlgren C, Karlsson A. Respiratory Burst in Human Neutrophils. J Immunol Methods (1999) 232:3-14. doi: 10.1016/S0022-1759(99)00146-5

46. Marin DP, Bolin AP, de Cássia Santos Macedo R, Sampaio SC, Otton R. ROS Production in Neutrophils From Alloxan-Induced Diabetic Rats Treated In Vivo With Astaxanthin. Int Immunopharmacol (2011) 11:103-9. doi: 10.1016/j.intimp.2010.10.013

47. Dupré-crochet S, Erard M, Nüße O. ROS Production in Phagocytes: Why, When, and Where? J Leukoc Biol (2013) 94:657-70. doi: 10.1189/jlb.1012544

48. Brinkmann V, Reichard U, Goosmann C, Fauler B, Uhlemann Y, Weiss DS, et al. Neutrophil Extracellular Traps Kill Bacteria. Science (80- ) (2004) 303:1532-5. doi: 10.1126/science. 1092385

49. Papayannopoulos V. Neutrophil Extracellular Traps in Immunity and Disease. Nat Rev Immunol (2018) 18:134-47. doi: 10.1038/nri.2017.105

50. Clark SR, Ma AC, Tavener SA, McDonald B, Goodarzi Z, Kelly MM, et al. Platelet TLR4 Activates Neutrophil Extracellular Traps to Ensnare Bacteria in Septic Blood. Nat Med (2007) 13:463-9. doi: 10.1038/nm1565

51. Espinosa V, Jhingran A, Dutta O, Kasahara S, Donnelly R, Du P, et al. Inflammatory Monocytes Orchestrate Innate Antifungal Immunity in the Lung. PLoS Pathog (2014) 10:1-13. doi: 10.1371/journal.ppat.1003940

52. Hall LJ, Murphy CT, Quinlan A, Hurley G, Shanahan F, Nally K, et al. Natural Killer Cells Protect Mice From DSS-induced Colitis by Regulating Neutrophil Function Via the NKG2A Receptor. Mucosal Immunol (2013) 6:1016-26. doi: $10.1038 / \mathrm{mi} .2012 .140$

53. Saffarzadeh M, Preissner KT. Fighting Against the Dark Side of Neutrophil Extracellular Traps in Disease: Manoeuvres for Host Protection. Curr Opin Hematol (2013) 20:3-9. doi: 10.1097/MOH.0b013e32835a0025

54. Luo L, Zhang S, Wang Y, Rahman M, Syk I, Zhang E, et al. Proinflammatory Role of Neutrophil Extracellular Traps in Abdominal Sepsis. Am J Physiol Lung Cell Mol Physiol (2014) 307:L586-96. doi: 10.1152/ajplung.00365.2013

55. Wan R, Jiang J, Hu C, Chen X, Chen C, Zhao B, et al. Neutrophil Extracellular Traps Amplify Neutrophil Recruitment and Inflammation in Neutrophilic Asthma by Stimulating the Airway Epithelial Cells to Activate the TLR4/NF-KB Pathway and Secrete Chemokines. Aging (Albany NY) (2020) 12:16820-36. doi: 10.18632/AGING.103479

56. Lorne E, Zmijewski JW, Zhao X, Liu G, Tsuruta Y, Park YJ, et al. Role of Extracellular Superoxide in Neutrophil Activation: Interactions Between Xanthine Oxidase and TLR4 Induce Proinflammatory Cytokine Production. Am J Physiol Cell Physiol (2008) 294:C985-93. doi: 10.1152/ ajpcell.00454.2007

57. Mayer-Scholl A, Averhoff P, Zychlinsky A. How Do Neutrophils and Pathogens Interact? Curr Opin Microbiol (2004) 7:62-6. doi: 10.1016/ j.mib.2003.12.004

58. Branzk N, Lubojemska A, Hardison SE, Wang Q, Gutierrez MG, Brown GD, et al. Neutrophils Sense Microbe Size and Selectively Release Neutrophil Extracellular Traps in Response to Large Pathogens. Nat Immunol (2014) 15:1017-25. doi: 10.1038/ni.2987

59. Farrera C, Fadeel B. Macrophage Clearance of Neutrophil Extracellular Traps is a Silent Process. J Immunol (2013) 191:2647-56. doi: 10.4049/ jimmunol.1300436

60. Kahlenberg JM, Carmona-Rivera C, Smith CK, Kaplan MJ. Neutrophil Extracellular Trap-Associated Protein Activation of the NLRP3 Inflammasome is Enhanced in Lupus Macrophages. J Immunol (2013) 190:1217-26. doi: 10.4049/jimmunol.1202388

61. Uderhardt S, Martins AJ, Tsang JS, Lämmermann T, Germain RN. Resident Macrophages Cloak Tissue Microlesions to Prevent Neutrophil-Driven Inflammatory Damage. Cell (2019) 177:541-55.e17. doi: 10.1016/ j.cell.2019.02.028

62. Rosowski EE, Raffa N, Knox BP, Golenberg N, Keller NP, Huttenlocher A. Macrophages Inhibit Aspergillus Fumigatus Germination and NeutrophilMediated Fungal Killing. PLoS Pathog (2018) 14:1-28. doi: 10.1371/ journal.ppat.1007229 
63. Afonso A, Silva J, Lousada S, Ellis AE, Silva MT. Uptake of Neutrophils and Neutrophilic Components by Macrophages in the Inflamed Peritoneal Cavity of Rainbow Trout (Oncorhynchus Mykiss). Fish Shellfish Immunol (1998) 8:319-38. doi: 10.1006/fsim.1998.0139

64. Jitkaew S, Witasp E, Zhang S, Kagan VE, Fadeel B. Induction of Caspaseand Reactive Oxygen Species-Independent Phosphatidylserine Externalization in Primary Human Neutrophils: Role in Macrophage Recognition and Engulfment. J Leukoc Biol (2009) 85:427-37. doi: $10.1189 / \mathrm{jlb} .0408232$

65. Cooper D, Lindberg FP, Gamble JR, Brown EJ, Vadas MA. Transendothelial Migration of Neutrophils Involves Integrin-Associated Protein (CD47). Proc Natl Acad Sci USA (1995) 92:3978-82. doi: 10.1073/pnas.92.9.3978

66. Smith WB, Gamble JR, Clark-Lewis I, Vadas MA. Interleukin-8 Induces Neutrophil Transendothelial Migration. Immunology (1991) 72:65-72.

67. Liu Y, Merlin D, Burst SL, Pochet M, Madara JL, Parkos CA. The Role of CD47 in Neutrophil Transmigration: Increased Rate of Migration Correlates With Increased Cell Surface Expression of CD47. J Biol Chem (2001) 276:40156-66. doi: 10.1074/jbc.M104138200

68. Wozniok I, Hornbach A, Schmitt C, Frosch M, Einsele H, Hube B, et al. Induction of ERK-kinase Signalling Triggers Morphotype-Specific Killing of Candida Albicans Filaments by Human Neutrophils. Cell Microbiol (2008) 10:807-20. doi: 10.1111/j.1462-5822.2007.01086.x

69. Bai M, Grieshaber-Bouyer R, Wang J, Schmider AB, Wilson ZS, Zeng L, et al. CD177 Modulates Human Neutrophil Migration Through ActivationMediated Integrin and Chemoreceptor Regulation. Blood (2017) 130:2092-100. doi: 10.1182/blood-2017-03-768507

70. Woodman RC, Reinhardt PH, Kanwar S, Johnston FL, Kubes P. Effects of Human Neutrophil Elastase (HNE) on Neutrophil Function In Vitro and in Inflamed Microvessels. Blood (1993) 82:2188-95. doi: 10.1182/ blood.v82.7.2188.bloodjournal8272188

71. Saito H, Minamiya Y, Saito S, Ogawa J. Endothelial Rho and Rho Kinase Regulate Neutrophil Migration Via Endothelial Myosin Light Chain Phosphorylation. J Leukoc Biol (2002) 72:829-36. doi: 10.1189/jlb.72.4.829

72. Issekutz AC, Rowter D, Springer TA. Role of ICAM-1 and ICAM-2 and Alternate CD11/CD18 Ligands in Neutrophil Transendothelial Migration. J Leukoc Biol (1999) 65:117-26. doi: 10.1002/jlb.65.1.117

73. Marshall LJ, Ramdin LSP, Brooks T, DPhil PC, Shute JK. Plasminogen Activator Inhibitor-1 Supports Il-8-Mediated Neutrophil Transendothelial Migration by Inhibition of the Constitutive Shedding of Endothelial IL-8/ Heparan Sulfate/Syndecan-1 Complexes. J Immunol (2003) 171:2057-65. doi: 10.4049/jimmunol.171.4.2057

74. Wang J, Xu J, Zhao X, Xie W, Wang H, Kong H. Fasudil Inhibits NeutrophilEndothelial Cell Interactions by Regulating the Expressions of GRP78 and BMPR2. Exp Cell Res (2018) 365:97-105. doi: 10.1016/j.yexcr.2018.02.026

75. Hu N, Westra J, Rutgers A, Doornbos-Van der Meer B, Huitema MG, Stegeman CA, et al. Decreased CXCR1 and CXCR2 Expression on Neutrophils in Anti-Neutrophil Cytoplasmic Autoantibody-Associated Vasculitides Potentially Increases Neutrophil Adhesion and Impairs Migration. Arthritis Res Ther (2011) 13:1-11. doi: 10.1186/ar3534

76. Gomez-Cambronero J, Horn J, Paul CC, Baumann MA. GranulocyteMacrophage Colony-Stimulating Factor is a Chemoattractant Cytokine for Human Neutrophils: Involvement of the Ribosomal P70 S6 Kinase Signaling Pathway. J Immunol (2003) 171:6846-55. doi: 10.4049/ jimmunol.171.12.6846

77. Chen Y, Corriden R, Inoue Y, Yip L, Hashiguchi N, Zinkernagel A, et al. ATP Release Guides Neutrophil Chemotaxis Via P2Y2 and A3 Receptors. Science (80- ) (2006) 314:1792-5. doi: 10.1126/science.1132559

78. Hennigan SM, Wang JH, Redmond HP, Bouchier-Hayes D. Neutrophil Heat Shock Protein Expression and Activation Correlate With Increased Apoptosis Following Transmigration Through the Endothelial Barrier. Shock (1999) 12:32-8. doi: 10.1097/00024382-199907000-00005

79. Moreland JG, Bailey G, Nauseef WM, Weiss JP. Organism-Specific Neutrophil-Endothelial Cell Interactions in Response to Escherichia Coli, Streptococcus Pneumoniae, and Staphylococcus Aureus. J Immunol (2004) 172:426-32. doi: 10.4049/jimmunol.172.1.426

80. Lauridsen HM, Gonzalez AL. Biomimetic, Ultrathin and Elastic Hydrogels Regulate Human Neutrophil Extravasation Across Endothelial-Pericyte Bilayers. PLoS One (2017) 12:1-19. doi: 10.1371/journal.pone.0171386
81. Lauridsen HM, Pellowe AS, Ramanathan A, Liu R, Miller-Jensen K, McNiff JM, et al. Tumor Necrosis Factor- $\alpha$ and IL-17A Activation Induces PericyteMediated Basement Membrane Remodeling in Human Neutrophilic Dermatoses. Am J Pathol (2017) 187:1893-906. doi: 10.1016/ j.ajpath.2017.04.008

82. Ueki H, Wang IH, Fukuyama S, Katsura H, Da Silva Lopes TJ, Neumann G, et al. In Vivo Imaging of the Pathophysiological Changes and Neutrophil Dynamics in Influenza Virus-Infected Mouse Lungs. Proc Natl Acad Sci USA (2018) 115:E6622-9. doi: 10.1073/pnas.1806265115

83. Barros-Becker F, Lam PY, Fisher R, Huttenlocher A. Live Imaging Reveals Distinct Modes of Neutrophil and Macrophage Migration Within Interstitial Tissues. J Cell Sci (2017) 130:3801-8. doi: 10.1242/jcs.206128

84. Tauzin S, Starnes TW, Becker FB, Lam Py, Huttenlocher A. Redox and Src Family Kinase Signaling Control Leukocyte Wound Attraction and Neutrophil Reverse Migration. J Cell Biol (2014) 207:589-98. doi: 10.1083/ jcb. 201408090

85. Colom B, Bodkin JV, Beyrau M, Woodfin A, Ody C, Rourke C, et al. Leukotriene B4-neutrophil Elastase Axis Drives Neutrophil Reverse Transendothelial Cell Migration In Vivo. Immunity (2015) 42:1075-86. doi: 10.1016/j.immuni.2015.05.010

86. Hellebrekers P, Vrisekoop N, Koenderman L. Neutrophil Phenotypes in Health and Disease. Eur J Clin Invest (2018) 48:1-9. doi: 10.1111/eci.12943

87. Yamahashi Y, Cavnar PJ, Hind LE, Berthier E, Bennin DA, Beebe D, et al. Integrin Associated Proteins Differentially Regulate Neutrophil Polarity and Directed Migration in 2D and 3D. BioMed Microdevices (2015) 17:1-9. doi: 10.1007/s10544-015-9998-x

88. Hamza B, Irimia D. Whole Blood Human Neutrophil Trafficking in a Microfluidic Model of Infection and Inflammation. Lab Chip (2015) 15:2625-33. doi: 10.1039/c5lc00245a

89. Hamza B, Wong E, Patel S, Cho H, Martel J, Irimia D. Retrotaxis of Human Neutrophils During Mechanical Confinement Inside Microfluidic Channels. Integr Biol (2014) 6:175-83. doi: 10.1039/c3ib40175h

90. Aranyosi AJ, Wong EA, Irimia D. A Neutrophil Treadmill to Decouple Spatial and Temporal Signals During Chemotaxis. Lab Chip (2015) 15:54956. doi: $10.1039 / \mathrm{c} 4 \mathrm{lc} 00970 \mathrm{c}$

91. Boribong BP, Lenzi MJ, Li L, Jones CN. Super-Low Dose Lipopolysaccharide Dysregulates Neutrophil Migratory Decision-Making. Front Immunol (2019) 10:359. doi: 10.3389/fimmu.2019.00359

92. Moussavi-Harami SF, Mladinich KM, Sackmann EK, Shelef MA, Starnes TW, Guckenberger DJ, et al. Microfluidic Device for Simultaneous Analysis of Neutrophil Extracellular Traps and Production of Reactive Oxygen Species. Integr Biol (United Kingdom) (2016) 8:243-52. doi: 10.1039/ c5ib00225g

93. Otawara M, Roushan M, Wang X, Ellett F, Yu YM, Irimia D. Microfluidic Assay Measures Increased Neutrophil Extracellular Traps Circulating in Blood After Burn Injuries. Sci Rep (2018) 8:1-9. doi: 10.1038/s41598-01834952-0

94. Tay HM, Dalan R, Li KHH, Boehm BO, Hou HW. A Novel Microdevice for Rapid Neutrophil Purification and Phenotyping in Type 2 Diabetes Mellitus. Small (2018) 14:1-10. doi: 10.1002/smll.201702832

95. Soroush F, Zhang T, King DJ, Tang Y, Deosarkar S, Prabhakarpandian B, et al. A Novel Microfluidic Assay Reveals a Key Role for Protein Kinase C $\delta$ in Regulating Human Neutrophil-Endothelium Interaction. J Leukoc Biol (2016) 100:1027-35. doi: 10.1189/jlb.3ma0216-087r

96. Jones CN, Dimisko L, Forrest K, Judice K, Poznansky MC, Markmann JF, et al. Human Neutrophils are Primed by Chemoattractant Gradients for Blocking the Growth of Aspergillus Fumigatus. J Infect Dis (2016) 213:46575. doi: 10.1093/infdis/jiv419

97. Hopke A, Scherer A, Kreuzburg S, Abers MS, Zerbe CS, Dinauer MC, et al. Neutrophil Swarming Delays the Growth of Clusters of Pathogenic Fungi. Nat Commun (2020) 11:1-15. doi: 10.1038/s41467-020-15834-4

98. Ellett F, Jalali F, Marand AL, Jorgensen J, Mutlu BR, Lee J, et al. Microfluidic Arenas for War Games Between Neutrophils and Microbes. Lab Chip (2019) 19:1205-16. doi: 10.1039/c8lc01263f

99. Lämmermann T, Bader BL, Monkley SJ, Worbs T, Wedlich-Söldner R, Hirsch K, et al. Rapid Leukocyte Migration by Integrin-Independent Flowing and Squeezing. Nature (2008) 453:51-5. doi: 10.1038/ nature 06887 
100. Wei Ling Lee S, Seager RJ, Litvak F, Spill F, Sieow JL, Leong PH, et al. Integrated in Silico and 3D In Vitro Model of Macrophage Migration in Response to Physical and Chemical Factors in the Tumor Microenvironment. Integr Biol (Camb) (2020) 12:90-108. doi: 10.1093/ intbio/zyaa007

101. Han S, Yan J-J, Shin Y, Jeon JS, Won J, Jeong HE, et al. A Versatile Assay for Monitoring In Vivo-Like Transendothelial Migration of Neutrophils. Lab Chip (2012) 12:3861-5. doi: 10.1039/c2lc40445a

102. Wu X, Newbold MA, Gao Z, Haynes CL. A Versatile Microfluidic Platform for the Study of Cellular Interactions Between Endothelial Cells and Neutrophils. Biochim Biophys Acta Gen Subj (2017) 1861:1122-30. doi: 10.1016/j.bbagen.2017.02.012

103. Huh D, Matthews BD, Mammoto A, Montoya-Zaavala M, Hsin HY, Ingber DE. Reconstituting Organ-Level Lung Functions on a Chip. Science (2010) 328:1662-8. doi: 10.1126/science.1188302

104. Benam KH, Villenave R, Lucchesi C, Varone A, Hubeau C, Lee HH, et al. Small Airway-on-a-Chip Enables Analysis of Human Lung Inflammation and Drug Responses In Vitro. Nat Methods (2016) 13:151-7. doi: 10.1038/ nmeth.3697

105. Wu X, Newbold MA, Haynes CL. Recapitulation of In Vivo-Like Neutrophil Transendothelial Migration Using a Microfluidic Platform. Analyst (2015) 140:5055-64. doi: 10.1039/C5AN00967G

106. Bischel LL, Sung KE, Jiménez-Torres JA, Mader B, Keely PJ, Beebe DJ. The Importance of Being a Lumen. FASEB J (2014) 28:4583-90. doi: 10.1096/ fj. $13-243733$

107. Polacheck WJ, Kutys ML, Tefft JB, Chen CS. Microfabricated blood vessels for modeling the vascular transport barrier. Nat Protoc (2019) 14 (5):142554. doi: 10.1038/s41596-019-0144-8

108. Chrobak KM, Potter DR, Tien J. Formation of Perfused, Functional Microvascular Tubes In Vitro. Microvasc Res (2006) 71:185-96. doi: 10.1016/j.mvr.2006.02.005

109. McGuigan AP, Sefton MV. Vascularized Organoid Engineered by Modular Assembly Enables Blood Perfusion. Proc Natl Acad Sci USA (2006) 103:11461-6. doi: 10.1073/pnas.0602740103

110. Price GM, Wong KHK, Truslow JG, Leung AD, Acharya C, Tien J. Effect of Mechanical Factors on the Function of Engineered Human Blood Microvessels in Microfluidic Collagen Gels. Biomaterials (2010) 31:6182-9. doi: 10.1016/j.biomaterials.2010.04.041

111. Brassard-Jollive N, Monnot C, Muller L, Germain S. In Vitro 3D Systems to Model Tumor Angiogenesis and Interactions With Stromal Cells. Front Cell Dev Biol (2020) 8:594903. doi: 10.3389/fcell.2020.594903

112. Tourovskaia A, Fauver M, Kramer G, Simonson S, Neumann T. TissueEngineered Microenvironment Systems for Modeling Human Vasculature. Exp Biol Med (2014) 239:1264-71. doi: 10.1177/1535370214539228

113. Bischel LL, Lee SH, Beebe DJ. A Practical Method for Patterning Lumens Through ECM Hydrogels Via Viscous Finger Patterning. J Lab Autom (2012) 17:96-103. doi: 10.1177/2211068211426694

114. Jiménez-Torres JA, Peery SL, Sung KE, Beebe DJ. Lumenext: A Practical Method to Pattern Luminal Structures in ECM Gels. Adv Healthc Mater (2016) 5:198-204. doi: 10.1002/adhm.201500608

115. Barkal LJ, Procknow CL, Álvarez-Garciá YR, Niu M, Jiménez-Torres JA, Brockman-Schneider RA, et al. Microbial Volatile Communication in Human Organotypic Lung Models. Nat Commun (2017) 8:1-10. doi: 10.1038/s41467-017-01985-4

116. Hind LE, Ingram PN, Beebe DJ, Huttenlocher A. Interaction With an Endothelial Lumen Increases Neutrophil Lifetime and Motility in Response to P Aeruginosa. Blood (2018) 132:1818-28. doi: 10.1182/blood2018-05-848465

117. Hind LE, Giese M, Schoen TJ, Beebe DJ, Keller N, Huttenlocher A. Immune Cell Paracrine Signaling Drives the Neutrophil Response to A. Fumigatus in an Infection-on-a-Chip Model. Cell Mol Bioeng (2021) 14(2):133-45. doi: 10.1007/s12195-020-00655-8

118. Yu J, Berthier E, Craig A, de Groot TE, Sparks S, Ingram PN, et al. Reconfigurable Open Microfluidics for Studying the Spatiotemporal Dynamics of Paracrine Signalling. Nat BioMed Eng (2019) 3:830-41. doi: 10.1038/s41551-019-0421-4

119. McMinn PH, Hind LE, Huttenlocher A, Beebe DJ. Neutrophil Trafficking on-a-Chip: An In Vitro, Organotypic Model for Investigating Neutrophil
Priming, Extravasation, and Migration With Spatiotemporal Control. Lab Chip (2019) 19:3697-705. doi: 10.1039/c9lc00562e

120. Bellan LM, Pearsall M, Cropek DM, Langer R. A 3D Interconnected Microchannel Network Formed in Gelatin by Sacrificial Shellac Microfibers. Adv Mater (2012) 24:5187-91. doi: 10.1002/adma.201200810

121. Lee JB, Wang X, Faley S, Baer B, Balikov DA, Sung HJ, et al. Development of 3D Microvascular Networks Within Gelatin Hydrogels Using Thermoresponsive Sacrificial Microfibers. Adv Healthc Mater (2016) 5:781-5. doi: 10.1002/adhm.201500792

122. Grigoryan B, Paulsen SJ, Corbett DC, Sazer DW, Fortin CL, Zaita AJ, et al. Multivascular Networks and Functional Intravascular Topologies Within Biocompatible Hydrogels. Science (80- ) (2019) 364:458-64. doi: 10.1126/ science.aav 9750

123. Sackmann EK, Berthier E, Young EWK, Shelef MA, Wernimont SA, Huttenlocher A, et al. Microfluidic Kit-on-a-Lid: A Versatile Platform for Neutrophil Chemotaxis Assays. Blood (2012) 120:45-53. doi: 10.1182/blood2012-03-416453

124. Berthier E, Guckenberger DJ, Cavnar P, Huttenlocher A, Keller NP, Beebe DJ. Kit-On-A-Lid-Assays for Accessible Self-Contained Cell Assays. Lab Chip (2013) 13:424-31. doi: 10.1039/c2lc41019b

125. Muldur S, Marand AL, Ellett F, Irimia D. "Measuring Spontaneous Neutrophil Motility Signatures From a Drop of Blood Using Microfluidics,". In: Methods in Cell Biology. Academic Press Inc. (2018). p. 93-107. doi: 10.1016/bs.mcb.2018.07.005

126. Wang X, Irimia D. "Neutrophil Chemotaxis in One Droplet of Blood Using Microfluidic Assays," A Gautreau, editor In: Cell Migration: Methods and Protocols. New York, NY: Springer New York. (2018). p. 351-60. doi: 10.1007/978-1-4939-7701-7_25

127. Jones CN, Hoang AN, Dimisko L, Hamza B, Martel J, Irimia D. Microfluidic Platform for Measuring Neutrophil Chemotaxis From Unprocessed Whole Blood. J Vis Exp (2014) 88:51215. doi: 10.3791/51215

128. Sackmann EKH, Berthier E, Schwantes EA, Fichtinger PS, Evans MD, Dziadzio LL, et al. Characterizing Asthma From a Drop of Blood Using Neutrophil Chemotaxis. Proc Natl Acad Sci USA (2014) 111:5813-8. doi: 10.1073/pnas.1324043111

129. Ellett F, Jorgensen J, Marand AL, Liu YM, Martinez MM, Sein V, et al. Diagnosis of Sepsis From a Drop of Blood by Measurement of Spontaneous Neutrophil Motility in a Microfluidic Assay. Nat BioMed Eng (2018) 2:20714. doi: 10.1038/s41551-018-0208-z

130. Raymond SL, Hawkins RB, Stortz JA, Murphy TJ, Ungaro R, Dirain ML, et al. Sepsis is Associated With Reduced Spontaneous Neutrophil Migration Velocity in Human Adults. PLoS One (2018) 13:1-12. doi: 10.1371/ journal.pone.0205327

131. Stroka KM, Aranda-Espinoza H. Endothelial Cell Substrate Stiffness Influences Neutrophil Transmigration Via Myosin Light Chain KinaseDependent Cell Contraction. Blood (2011) 118:1632-40. doi: 10.1182/ blood-2010-11-321125

132. Stroka KM, Levitan I, Aranda-Espinoza H. OxLDL and Substrate Stiffness Promote Neutrophil Transmigration by Enhanced Endothelial Cell Contractility and ICAM-1. J Biomech (2012) 45:1828-34. doi: 10.1016/ j.jbiomech.2012.04.011

133. Stroka KM, Hayenga HN, Aranda-Espinoza H. Human Neutrophil Cytoskeletal Dynamics and Contractility Actively Contribute to Trans-Endothelial Migration. PLoS One (2013) 8:1-11. doi: 10.1371/journal.pone.0061377

134. Lamberti G, Prabhakarpandian B, Garson C, Smith A, Pant K, Wang B, et al. Bioinspired Microfluidic Assay for In Vitro Modeling of LeukocyteEndothelium Interactions. Anal Chem (2014) 86:8344-51. doi: 10.1021/ ac5018716

135. Ayres-Sander CE, Lauridsen H, Maier CL, Sava P, Pober JS, Gonzalez AL. Transendothelial Migration Enables Subsequent Transmigration of Neutrophils Through Underlying Pericytes. PLoS One (2013) 8:1-12. doi: 10.1371/journal.pone.0060025

136. Lauridsen HM, Pober JS, Gonzalez AL. A Composite Model of the Human Postcapillary Venule for Investigation of Microvascular Leukocyte Recruitment. FASEB J (2014) 28:1166-80. doi: 10.1096/fj.13-240986

137. Pellowe AS, Sauler M, Hou Y, Merola J, Liu R, Calderon B, et al. Endothelial Cell-Secreted MIF Reduces Pericyte Contractility and Enhances Neutrophil Extravasation. FASEB J (2019) 33:2171-86. doi: 10.1096/fj.201800480R 
138. Sava P, Cook IO, Mahal RS, Gonzalez AL. Human Microvascular Pericyte Basement Membrane Remodeling Regulates Neutrophil Recruitment. Microcirculation (2015) 22:54-67. doi: 10.1111/micc.12173

139. Kwak BS, Jin SP, Kim SJ, Kim EJ, Chung JH, Sung JH. Microfluidic Skin Chip With Vasculature for Recapitulating the Immune Response of the Skin Tissue. Biotechnol Bioeng (2020) 117:1-11. doi: 10.1002/bit.27320

140. Mejías JC, Nelson MR, Liseth O, Roy K. A 96-Well Format Microvascularized Human lung-on-A-chip Platform for Microphysiological Modeling of Fibrotic Diseases. Lab Chip (2020) 20:3601-11. doi: 10.1039/d0lc00644k

141. Wang X, Hossain M, Bogoslowski A, Kubes P, Irimia D. Chemotaxing Neutrophils Enter Alternate Branches At Capillary Bifurcations. Nat Commun (2020) 11:1-12. doi: 10.1038/s41467-020-15476-6

142. Reátegui E, Jalali F, Khankhel AH, Wong E, Cho H, Lee J, et al. Microscale Arrays for the Profiling of Start and Stop Signals Coordinating HumanNeutrophil Swarming. Nat BioMed Eng (2017) 1:1-12. doi: 10.1038/s41551017-0094

143. Hopke A, Irimia D. "Ex Vivo Human Neutrophil Swarming Against Live Microbial Targets". In: M Quinn and F DeLeo, editors. Neutrophil. Methods in Molecular Biology. New York, NY: Humana. (2020). p. 107-16.

144. Walters N, Nguyen LTH, Zhang J, Shankaran A, Reátegui E. Extracellular Vesicles as Mediators of: In Vitro Neutrophil Swarming on a Large-Scale Microparticle Array. Lab Chip (2019) 19:2874-84. doi: 10.1039/ c9lc00483a

145. Tang FSM, Hansbro PM, Burgess JK, Ammit AJ, Baines KJ, Oliver BG. A Novel Immunomodulatory Function of Neutrophils on rhinovirus-Activated Monocytes In Vitro. Thorax (2016) 71:1039-49. doi: 10.1136/thoraxjnl2015-207781

146. Kukulski F, Ben Yebdri F, Lefebvre J, Warny M, Tessier PA, Sévigny J. Extracellular Nucleotides Mediate LPS-Induced Neutrophil Migration In Vitro and In Vivo. J Leukoc Biol (2007) 81:1269-75. doi: 10.1189/jlb.1206758

147. Yang D, Chen Q, Chertov O, Oppenheim JJ. Human Neutrophil Defensins Selectively Chemoattract Naive T and Immature Dendritic Cells. J Leukoc Biol (2000) 68:9-14.

148. Gafa V, Remoli ME, Giacomini E, Gagliardi MC, Lande R, Severa M, et al. In Vitro Infection of Human Dendritic Cells by Aspergillus Fumigatus Conidia Triggers the Secretion of Chemokines for Neutrophil and Th1 Lymphocyte Recruitment. Microbes Infect (2007) 9:971-80. doi: 10.1016/ j.micinf.2007.03.015

149. Pelletier M, Maggi L, Micheletti A, Lazzeri E, Tamassia N, Costantini C, et al. Evidence for a Cross-Talk Between Human Neutrophils and Th17 Cells. Blood (2010) 115:335-43. doi: 10.1182/blood-2009-04-216085

150. Ellett F, Jorgensen J, Frydman GH, Jones CN, Irimia D. Neutrophil Interactions Stimulate Evasive Hyphal Branching by Aspergillus Fumigatus. PLoS Pathog (2017) 13:1-22. doi: 10.1371/journal.ppat.1006154

151. Grundy JE, Lawson KM, MacCormac LP, Fletcher JM, Yong KL. Cytomegalovirus-Infected Endothelial Cells Recruit Neutrophils by the Secretion of C-X-C Chemokines and Transmit Virus by Direct Neutrophil- Endothelial Cell Contact and During Neutrophil Transendothelial Migration. J Infect Dis (1998) 177:1465-74. doi: 10.1086/ 515300

152. Deng Y, Herbert JA, Smith CM, Smyth RL. An In Vitro Transepithelial Migration Assay to Evaluate the Role of Neutrophils in Respiratory Syncytial Virus (RSV) Induced Epithelial Damage. Sci Rep (2018) 8:1-12. doi: 10.1038/ s41598-018-25167-4

153. Craigen JL, Yong KL, Jordan NJ, Maccormac LP, Westwick J, Akbar AN, et al. Human Cytomegalovirus Infection Up-Regulates Interleukin-8 Gene Expression and Stimulates Neutrophil Transendothelial Migration. Immunology (1997) 92:138-45. doi: 10.1046/j.1365-2567.1997.00310.x

154. Lamberti G, Soroush F, Smith A, Kiani MF, Prabhakarpandian B, Pant K. Adhesion Patterns in the Microvasculature are Dependent on Bifurcation Angle. Microvasc Res (2015) 99:19-25. doi: 10.1016/j.mvr.2015.02.004

155. Birbrair A, Zhang T, Wang ZM, Messi ML, Mintz A, Delbono O. Pericytes At the Intersection Between Tissue Regeneration and Pathology. Clin Sci (2015) 128:81-93. doi: 10.1042/CS20140278

156. Alimperti S, Mirabella T, Bajaj V, Polacheck W, Pirone DM, Duffield J, et al. Three-Dimensional Biomimetic Vascular Model Reveals a RhoA, Racl, and $\mathrm{N}$-cadherin Balance in Mural Cell-Endothelial Cell-Regulated Barrier
Function. Proc Natl Acad Sci USA (2017) 114:8758-63. doi: 10.1073/pnas. 1618333114

157. Vargas A, Roux-Dalvai F, Droit A, Lavoie JP. Neutrophil-Derived Exosomes: A New Mechanism Contributing to Airway Smooth Muscle Remodeling. Am J Respir Cell Mol Biol (2016) 55:450-61. doi: 10.1165/rcmb.2016-0033OC

158. Middleton SJ, Shorthouse M, Hunter JO. Relaxation of Distal Colonic Circular Smooth Muscle by Nitric Oxide Derived From Human Leucocytes. Gut (1993) 34:814-7. doi: 10.1136/gut.34.6.814

159. Vanaudenaerde BM, Wuyts WA, Dupont LJ, Van Raemdonck DE, Demedts MM, Verleden GM. Interleukin-17 Stimulates Release of Interleukin-8 by Human Airway Smooth Muscle Cells In Vitro: A Potential Role for Interleukin-17 and Airway Smooth Muscle Cells in Bronchiolitis Obliterans Syndrome. J Heart Lung Transplant (2003) 22:1280-3. doi: 10.1016/S1053-2498(02)01234-2

160. Humayun M, Chow CW, Young EWK. Microfluidic Lung Airway-on-aChip With Arrayable Suspended Gels for Studying Epithelial and Smooth Muscle Cell Interactions. Lab Chip (2018) 18:1298-309. doi: 10.1039/ c7lc01357d

161. Costa-Almeida R, Soares R, Granja PL. Fibroblasts as Maestros Orchestrating Tissue Regeneration. J Tissue Eng Regener Med (2018) 12:240-51. doi: 10.1002/ term. 2405

162. Jordana M, Sarnstrand B, Sime PJ, Ramis I. Immune-Inflammatory Functions of Fibroblasts. Eur Respir J (1994) 7:2212-22. doi: 10.1183/ 09031936.94 .07122212

163. Smith RS, Smith TJ, Blieden TM, Phipps RP. Fibroblasts as Sentinel CellsSynthesis of Chemokines and Regulation of Inflammation. Am J Pathol (1997) 151:317-22.

164. Takashiba S, Takigawa M, Takahashi K, Myokai F, Nishimura F, Chihara T, et al. Interleukin-8 is a Major Neutrophil Chemotactic Factor Derived From Cultured Human Gingival Fibroblasts Stimulated With Interleukin-1 $\beta$ or Tumor Necrosis Factor Alpha. Infect Immun (1992) 60:5253-8. doi: 10.1128/ iai.60.12.5253-5258.1992

165. Lally F, Smith E, Filer A, Stone MA, Shaw JS, Nash GB, et al. A Novel Mechanism of Neutrophil Recruitment in a Coculture Model of the Rheumatoid Synovium. Arthritis Rheumatol (2005) 52:3460-9. doi: 10.1002/art.21394

166. Tavares NM, Araújo-Santos T, Afonso L, Nogueira PM, Lopes UG, Soares RP, et al. Understanding the Mechanisms Controlling Leishmania Amazonensis Infection In Vitro: The Role of LTB4 Derived From Human Neutrophils. J Infect Dis (2014) 210:656-66. doi: 10.1093/infdis/jiu158

167. Sun D, Shi M. Neutrophil Swarming Toward Cryptococcus Neoformans is Mediated by Complement and Leukotriene B4. Biochem Biophys Res Commun (2016) 477:945-51. doi: 10.1016/j.bbrc.2016.07.005

168. Geissmann F, Manz MG, Jung S, Sieweke MH, Merad M, Ley K. Development of Monocytes, Macrophages, and Dendritic Cells. Science (80- ) (2010) 327:656-61. doi: 10.1126/science.1178331

169. Serbina N, Jia T, Hohl TM, Pamer EG. Monocyte-Mediated Defense Against Microbial Pathogens. Annu Rev Immunol (2008) 26:421-52. doi: 10.1146/ annurev.immunol.26.021607.090326

170. Zhang YG, Song Y, Guo XL, Miao RY, Fu YQ, Miao CF, et al. Exosomes Derived From oxLDL-Stimulated Macrophages Induce Neutrophil Extracellular Traps to Drive Atherosclerosis. Cell Cycle (2019) 18:2672-82. doi: 10.1080/15384101.2019.1654797

171. Novais FO, Santiago RC, Báfica A, Khouri R, Afonso L, Borges VM, et al. Neutrophils and Macrophages Cooperate in Host Resistance Against Leishmania Braziliensis Infection. J Immunol (2009) 183:8088-98. doi: 10.4049/jimmunol.0803720

172. Barrientos L, Bignon A, Gueguen C, de Chaisemartin L, Gorges R, Sandré C, et al. Neutrophil Extracellular Traps Downregulate LipopolysaccharideInduced Activation of Monocyte-Derived Dendritic Cells. J Immunol (2014) 193:5689-98. doi: 10.4049/jimmunol.1400586

173. Deuel TF, Senior RM, Chang D, Griffin GL, Heinrikson RL, Kaiser ET. Platelet Factor 4 is Chemotactic for Neutrophils and Monocytes. Proc Natl Acad Sci USA (1981) 78:4584-7. doi: 10.1073/pnas.78.7.4584

174. Buttrum SM, Hatton R, Nash GB. Selectin-Mediated Rolling of Neutrophils on Immobilized Platelets. Blood (1993) 82:1165-74. doi: 10.1182/ blood.v82.4.1165.bloodjournal8241165 
175. Kuravi SJ, Harrison P, Rainger GE, Nash GB. Ability of Platelet-Derived Extracellular Vesicles to Promote Neutrophil-Endothelial Cell Interactions. Inflammation (2019) 42:290-305. doi: 10.1007/s10753-018-0893-5

176. Tamassia N, Arruda-Silva F, Wright HL, Moots RJ, Gardiman E, BianchettoAguilera F, et al. Human Neutrophils Activated Via TLR8 Promote Th17 Polarization Through IL-23. J Leukoc Biol (2019) 105:1155-65. doi: 10.1002/ JLB.MA0818-308R

177. Enoksson M, Möller-Westerberg C, Wicher G, Fallon PG, Forsberg-Nilsson $\mathrm{K}$, Lunderius-Andersson C, et al. Intraperitoneal Influx of Neutrophils in Response to IL-33 is Mast Cell-Dependent. Blood (2013) 121:530-6. doi: 10.1182/blood-2012-05-434209

178. Malaviya R, Abraham SN. Role of Mast Cell Leukotrienes in Neutrophil Recruitment and Bacterial Clearance in Infectious Peritonitis. J Leukoc Biol (2000) 67:841-6. doi: 10.1002/jlb.67.6.841

179. Malaviya R, Gao Z, Thankavel K, Van der Merwe PA, Abraham SN. The Mast Cell Tumor Necrosis Factor $\alpha$ Response to FimH-Expressing Escherichia Coli is Mediated by the GlycosylphosphatidylinositolAnchored Molecule CD48. Proc Natl Acad Sci USA (1999) 96:8110-5. doi: 10.1073 /pnas.96.14.8110

180. Costantini C, Micheletti A, Calzetti F, Perbellini O, Pizzolo G, Cassatella MA. Neutrophil Activation and Survival are Modulated by Interaction With NK Cells. Int Immunol (2010) 22:827-38. doi: 10.1093/intimm/dxq434

181. Thorén FB, Riise RE, Ousbäck J, Della Chiesa M, Alsterholm M, Marcenaro E, et al. Human NK Cells Induce Neutrophil Apoptosis Via an NKp46- and Fas-Dependent Mechanism. J Immunol (2012) 188:1668-74. doi: 10.4049/ jimmunol.1102002

182. Robinet P, Baychelier F, Fontaine T, Picard C, Debré P, Vieillard V, et al. A Polysaccharide Virulence Factor of a Human Fungal Pathogen Induces Neutrophil Apoptosis Via NK Cells. J Immunol (2014) 192:5332-42. doi: 10.4049/jimmunol.1303180
183. Gelzer J, Suter E. Metabolic Interactions In Vitro Between Polymorphonuclear Leukocytes and Pathogenic and Nonpathogenic Microorganisms. Experientia (1959) 15:417-9. doi: 10.1007/BF 02157686

184. Van De Veerdonk FL, Gresnigt MS, Romani L, Netea MG, Latgé JP. Aspergillus Fumigatus Morphology and Dynamic Host Interactions. Nat Rev Microbiol (2017) 15:661-74. doi: 10.1038/nrmicro.2017.90

185. Jones CN, Ellett F, Robertson AL, Forrest KM, Judice K, Balkovec JM, et al. Bifunctional Small Molecules Enhance Neutrophil Activities Against Aspergillus Fumigatus In Vivoand In Vitro. Front Immunol (2019) 10:644. doi: 10.3389/fimmu.2019.00644

186. Owen-Woods C, Joulia R, Barkaway A, Rolas L, Ma B, Nottebaum AF, et al. Local Microvascular Leakage Promotes Trafficking of Activated Neutrophils to Remote Organs. J Clin Invest (2020) 130:2301-18. doi: 10.1172/JCI133661

187. Woodfin A, Voisin M, Beyrau M, Colom B, Caille D, Diapouli F, et al. The Junctional Adhesion Molecule JAM-C Regulates Polarized Transendothelial Migration of Neutrophils In Vivo. Nat Immunol (2011) 12:761-70. doi: $10.1038 /$ ni.2062

Conflict of Interest: The authors declare that the research was conducted in the absence of any commercial or financial relationships that could be construed as a potential conflict of interest.

Copyright (c) 2021 Richardson, Calo and Hind. This is an open-access article distributed under the terms of the Creative Commons Attribution License (CC BY). The use, distribution or reproduction in other forums is permitted, provided the original author(s) and the copyright owner(s) are credited and that the original publication in this journal is cited, in accordance with accepted academic practice. No use, distribution or reproduction is permitted which does not comply with these terms. 